\title{
Search for Dark Matter and Large Extra Dimensions in $p p$ Collisions Yielding a Photon and Missing Transverse Energy
}

\author{
S. Chatrchyan et al. ${ }^{*}$ \\ (CMS Collaboration) \\ (Received 4 April 2012; published 27 June 2012)
}

\begin{abstract}
Results are presented from a search for new physics in the final state containing a photon $(\gamma)$ and missing transverse energy $\left(\mathscr{E}_{T}\right)$. The data correspond to an integrated luminosity of $5.0 \mathrm{fb}^{-1}$ collected in $p p$ collisions at $\sqrt{s}=7 \mathrm{TeV}$ by the CMS experiment. The observed event yield agrees with standardmodel expectations for the $\gamma+\not E_{T}$ events. Using models for the production of dark-matter particles $(\chi)$, we set $90 \%$ confidence level (C.L.) upper limits of 13.6-15.4 fb on $\chi$ production in the $\gamma+\not E_{T}$ state. These provide the most sensitive upper limits for spin-dependent $\chi$-nucleon scattering for $\chi$ masses $\left(M_{\chi}\right)$ between 1 and $100 \mathrm{GeV}$. For spin-independent contributions, the present limits are extended to $M_{\chi}<$ $3.5 \mathrm{GeV}$. For models with 3-6 large extra dimensions, our data exclude extra-dimensional Planck scales between 1.64 and $1.73 \mathrm{TeV}$ at $95 \%$ C.L.
\end{abstract}

DOI: 10.1103/PhysRevLett.108.261803

Final states in $p p$ collisions at the Large Hadron Collider (LHC), containing a photon $(\gamma)$ of large transverse momentum $\left(p_{T}\right)$ and missing transverse energy $\left(\mathbb{E}_{T}\right)$, are used to investigate two proposals of physics beyond the standard model (SM). One involves a model for dark matter (DM), which is now accepted as the dominant nonbaryonic contribution to the matter density of the Universe [1]. Direct searches for a DM candidate $(\chi)$ rely on detection through elastic $\chi$-nucleon scattering. Indirect searches consist of observation of photons or neutrinos produced in $\chi \bar{\chi}$ annihilations in astrophysical sources. At the LHC, DM can be produced in the reaction $q \bar{q} \rightarrow \gamma \chi \bar{\chi}$, where the photon is radiated by one of the incoming quarks. The final state is a high- $p_{T}$ photon and $\mathscr{E}_{T}$. Recent theoretical work [2-5] casts this process in terms of a massive mediator in the $s$ channel that couples to a $\chi \bar{\chi}$ pair of Dirac particles. This process is contracted into an effective theory with a contact interaction scale $\Lambda$, given by $\Lambda^{-2}=g_{\chi} g_{q} M_{M}^{-2}$, where $M_{M}$ is the mediator mass and $g_{\chi}$ and $g_{q}$ are its couplings to $\chi$ and quarks, respectively. The model provides a way to connect the $t$-channel $\chi$-nucleon elastic scattering to the $s$-channel pair-production mechanism. The effective $s$-channel operator can be chosen to represent either a vector or axialvector, spin-independent or spin-dependent interaction, respectively.

The $\gamma+\mathscr{E}_{T}$ final state also has sensitivity to models of extra spatial dimensions. The Arkani-Hamed, Dimopoulos, and Dvali model (ADD) [6], in particular, provides a

*Full author list given at the end of the article.

Published by the American Physical Society under the terms of the Creative Commons Attribution 3.0 License. Further distribution of this work must maintain attribution to the author(s) and the published article's title, journal citation, and DOI.
PACS numbers: $13.85 . \mathrm{Rm}, 11.25 . \mathrm{Wx}, 14.70 . \mathrm{Kv}, 14.80 . \mathrm{Nb}$

possible solution to the hierarchy problem, viz., the disparity between two fundamental scales of nature: the electroweak unification scale $\left(M_{\mathrm{EW}} \approx 100 \mathrm{GeV}\right)$ and the Planck scale $\left(M_{\mathrm{Pl}} \approx 10^{19} \mathrm{GeV}\right)$. In this framework, space-time is postulated to have $n$ extra compact spatial dimensions with a characteristic scale $R$, leading to a modified Planck scale, $M_{D}$, given by $M_{\mathrm{Pl}}^{2} \approx M_{D}^{n+2} R^{n}$. Assuming $M_{D}$ is of the same order as $M_{\mathrm{EW}}$, the observed large value of $M_{\mathrm{Pl}}$ can be interpreted as being a consequence of the "large" size of $R$ (relative to the Planck length $\approx M_{\mathrm{Pl}}^{-1}$ ) and the number of extra dimensions in the theory. The ADD model predicts the production of gravitons that appear as Kaluza-Klein (KK) modes, where momenta in the extra dimensions appear as observable massive states, except for the zero mode of the KK excitation, which corresponds to the massless graviton in $4+n$ dimensions. The process $q \bar{q} \rightarrow \gamma G$, where the graviton $G$ escapes detection, motivates the search for events with single high- $p_{T}$ isolated photons. While the individual $q G$ couplings are small, the number of expected KK graviton states is large enough to produce a measurable cross section, making it possible to discover large extra dimensions, or to set lower limits on $M_{D}$ as a function of $n$ and upper limits on the ADD cross section. The same physical phenomena can be accessed through the single-jet (monojet) production channel $[7,8]$.

This search uses data collected with the Compact Muon Solenoid (CMS) detector [9]. The momenta of charged particles are measured using a silicon pixel and strip tracker that is immersed in a $3.8 \mathrm{~T}$ superconducting solenoid, and covers the pseudorapidity range $|\eta|<2.5$. The pseudorapidity is $\eta=-\ln [\tan (\theta / 2)]$, where $\theta$ is the polar angle measured relative to the counterclockwise-beam direction. The tracker is surrounded by a crystal electromagnetic calorimeter (ECAL) and a brass-scintillator hadron calorimeter (HCAL). Both measure particle energy depositions 
and consist of a barrel assembly and two endcaps that provide coverage in the range of $|\eta|<3$.0. A quartz-fiber and steel Cherenkov forward detector extends the calorimetric coverage to $|\eta|<5$. Muons are measured in gas detectors embedded in the steel return yoke outside of the solenoid.

The primary background for the $\gamma+\mathbb{E}_{T}$ signal is the irreducible SM background from $Z \gamma \rightarrow \nu \bar{\nu} \gamma$ production. This and other SM backgrounds, including $W \gamma, W \rightarrow e \nu$, $\gamma+$ jet, multijet (referred to as QCD), and diphoton events, as well as backgrounds from beam halo and cosmic-ray muons, are taken into account in the analysis.

Events are selected from a data sample corresponding to an integrated luminosity of $5.0 \mathrm{fb}^{-1}$ collected using a twolevel trigger system, with Level-1 (L1) seeding high level triggers (HLT). The single-photon triggers comprising this search are not prescaled, and are fully efficient within the selected signal region of $\left|\eta^{\gamma}\right|<1.44$ [10] and $\left.p_{T}^{\gamma}\right\rangle$ $145 \mathrm{GeV}$. To optimize the analysis for single high- $p_{T}$ photons accompanied by large $\mathbb{E}_{T}$, photon candidates are restricted to be in the central barrel region, where purity is highest. To distinguish photon candidates from jets, we apply additional calorimetric selections. The ratio of energy deposited in the HCAL to that in the ECAL within a cone of $\Delta R=0.15$ is required to be less than 0.05 , where $\Delta R=\sqrt{(\Delta \phi)^{2}+(\Delta \eta)^{2}}$ is defined relative to the photon candidate and the azimuthal angle $\phi$ is measured in the plane perpendicular to the beam axis. Photon candidates must also have a shower distribution in the ECAL consistent with that expected for a photon [10].

Isolation requirements on photon candidates impose upper limits on the energy deposited in the detector around the axis defined by the EM cluster position and the primary vertex [10]. In particular, the scalar sum of $p_{T}$ depositions in the ECAL within a hollow cone of $0.06<\Delta R<0.40$, excluding depositions within $|\Delta \eta|=0.04$ of the cluster center, must be $<4.2 \mathrm{GeV}+0.006 \times p_{T}^{\gamma}$; the sum of scalar $p_{T}$ depositions in the HCAL within a hollow cone of $0.15<\Delta R<0.40$ must be $<2.2 \mathrm{GeV}+0.0025 \times p_{T}^{\gamma}$; and the scalar sum of track $p_{T}$ values in a hollow cone of $0.04<\Delta R<0.40$, excluding depositions that are closer to the cluster center than $|\Delta \eta|=0.015$, must be $<2.0 \mathrm{GeV}+0.001 \times p_{T}^{\gamma}$ (with $p_{T}$ in $\mathrm{GeV}$ units). The vetoes defined by the $|\Delta \eta|$ cutoffs are needed to maintain high efficiency for photons that initiate EM showers within the tracker. The tracker isolation requirement is based on tracks that originate from the primary vertex.

Since the high luminosity of the LHC yields multiple $p p$ interactions per bunch crossing, there are several reconstructed vertices per event. The primary vertex is defined as the vertex that corresponds to the largest sum of the squares of the associated track- $p_{T}$ values. However, to ensure that photon candidates are isolated from charged particle tracks in events with multiple vertices, the tracker isolation requirement must be passed by all reconstructed vertices, or the event is rejected.
The $\mathbb{E}_{T}$ is defined by the magnitude of the vector sum of the transverse energies of all of the reconstructed objects in the event, and is computed using a particle-flow algorithm [11]. The candidate events are required to have $\mathscr{E}_{T}>$ $130 \mathrm{GeV}$.

All events are required to have the energy deposited in the crystal containing the largest signal within the photon to be within \pm 3 ns of the time expected for particles from a collision. This requirement reduces instrumental background arising from showers induced by bremsstrahlung from muons in the beam halo or in cosmic rays. Spurious signals embedded within EM showers that otherwise pass selection criteria are eliminated by requiring consistency among the energy deposition times for all crystals within an electromagnetic shower. Photon candidates are removed if they are likely to be electrons, as inferred from characteristic patterns of hits in the pixel detector, called "pixel seeds," that are matched to the EM clusters [12]. In addition, a veto applied to events that contain muon candidates, including those that do not emanate from the collision point, prevents bremsstrahlung from muons in cosmic rays and the beam halo from being reconstructed as prompt photons balanced by $\mathscr{E}_{T}$. Finally, events are vetoed if they contain significant hadronic activity, defined by (i) a track with $p_{T}>20 \mathrm{GeV}$ that is $\Delta R>0.04$ away from the photon candidate, or (ii) a jet that is reconstructed with $p_{T}>$ $40 \mathrm{GeV}$ using the anti- $k_{T}$ [13] particle-flow algorithm [11], within $|\eta|<3.0$ and $\Delta R>0.5$ of the axis of the photon. After applying all of the selection criteria, 73 candidate events are found.

Backgrounds that are out of time with the collisions are estimated from data by examining the transverse distribution of energy in the EM cluster and the time of arrival of the signal in the crystal with the largest energy deposition. Templates for anomalous signals [14], cosmic-ray muons, and beam halo events are fitted to a candidate sample that has no timing requirement, which reveals that the only significant residual contribution to the in-time sample arises from halo muons, with an estimated 11.1 \pm 5.6 events.

Electrons misidentified as photons arise mainly from $W \rightarrow e \nu$ events. The matching of electron showers to pixel seeds has an efficiency of $\epsilon=0.9940 \pm 0.0025$, as estimated with Monte Carlo (MC) simulated events and verified with $Z \rightarrow e e$ events in data. Scaling a control sample of electron candidates by $(1-\epsilon) / \epsilon$ yields an estimated contribution of $3.5 \pm 1.5 \mathrm{~W} \rightarrow e \nu$ events in the candidate sample.

The contamination from jets misidentified as photons is estimated by using a control sample of EM-enriched QCD events to calculate the ratio of events that pass the signal photon criteria relative to those that pass looser photon criteria but fail an isolation requirement. Since the EMenriched sample also includes the production of direct single photons, this additional contribution to the ratio is 
estimated by fitting templates of energy-weighted shower widths from MC-simulated $\gamma+$ jets events to an independent QCD data sample, and used to subtract the $\gamma+$ jets contribution. This corrected ratio is applied to a subset of the EM-enriched jet events that passes loose photon identification and additional single-photon event selection criteria, providing a background contribution of $11.2 \pm 2.8$ jet events.

Backgrounds from $Z \gamma \rightarrow \nu \bar{\nu} \gamma, W \gamma \rightarrow \ell \nu \gamma, \gamma+$ jet, and diphoton events are estimated from MC samples processed through the full GEANT4-based simulation of the CMS detector $[15,16]$, trigger emulation and event reconstruction used for data. The $W \gamma \rightarrow \ell \nu \gamma$ samples are generated with MADGRAPH5 [17], and the cross section is corrected to include next-to-leading order (NLO) effects through a $K$ factor calculated with MCFM [18]. The $Z \gamma \rightarrow$ $\nu \bar{\nu} \gamma, \gamma+$ jet, and diphoton samples are obtained using the PYTHIA 6.424 generator [19] at leading order (LO) and CTEQ6L1 [20] parton distribution functions (PDF). The $Z \gamma \rightarrow \nu \bar{\nu} \gamma$ sample is also scaled up to reflect NLO contributions given in Ref. [21]. Good agreement between data and the rescaled MC prediction for the $Z \gamma \rightarrow \ell \ell \gamma$ channel has been obtained in previous CMS studies [22]. The uncertainty on $Z \gamma \rightarrow \nu \bar{\nu} \gamma$ and the other backgrounds takes into account several sources: theoretical uncertainties on the LO cross section and $K$ factors; the uncertainty on the scale factor that models the data-MC difference in the efficiency; and systematic uncertainties on the photonvertex assignment, modeling of pileup, and the accuracy of the energy calibration and resolution for photons, jets, and $\mathbb{E}_{T}$. The expected contribution from the $Z \gamma \rightarrow \nu \bar{\nu} \gamma$ process to the background is $45.3 \pm 6.9$ events. The combined expected background from $W \gamma \rightarrow \ell \nu \gamma, \gamma+$ jet, and diphoton events is $4.1 \pm 1.0$.

The 73 observed events in data agree with the total expected background of $75.1 \pm 9.5$ events. Distributions in photon $p_{T}$ for the selected candidate events and for those estimated from background are shown in Fig. 1. The spectra expected from ADD for $M_{D}=1 \mathrm{TeV}$ and $n=3$ are superimposed for comparison. Based on these results, exclusion limits are set for the DM and ADD models.

The limits on the cross sections are calculated by dividing the number of events in data less the predicted number of background events by the product $A \times \epsilon \times \mathcal{L}$, where $A$ is the geometric and kinematic acceptance of the selection criteria, $\epsilon$ is the selection efficiency for signal, and $\mathcal{L}$ is the integrated luminosity. $A \times \epsilon$ is factorized into $A \times \epsilon_{\mathrm{MC}}$, estimated from the $\mathrm{MC}$ events, multiplied by a scale factor (SF), to account for the difference in efficiency between the $\mathrm{MC}$ prediction and data.

The efficiency associated with the product $A \times \epsilon_{\mathrm{MC}}$ for the signal cross section for both models is determined from MC samples. For the model of DM, the MC samples are produced using a software package from Ref. [3], requiring

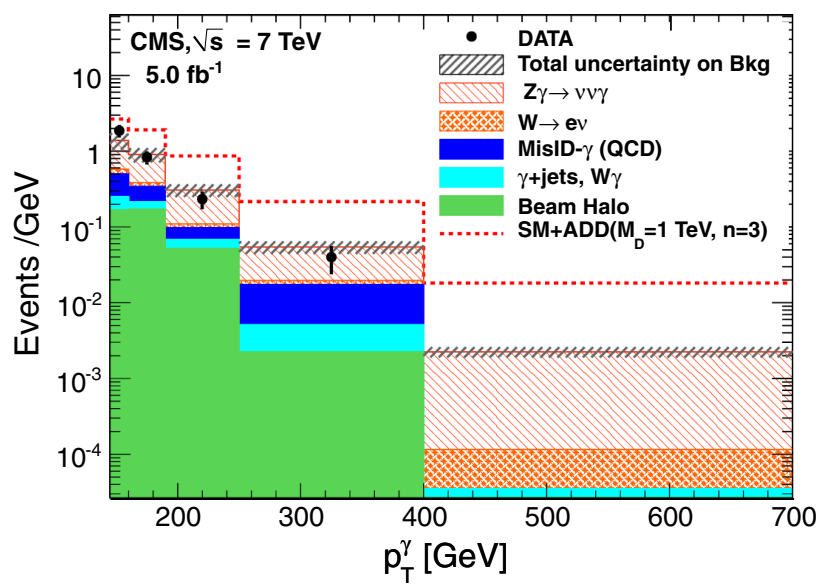

FIG. 1 (color online). The photon $p_{T}$ distribution for the candidate sample, compared with estimated contributions from $\mathrm{SM}$ backgrounds and a prediction from $\mathrm{ADD}$ for $M_{D}=1 \mathrm{TeV}$ and $n=3$.

$p_{T}^{\gamma}>125 \mathrm{GeV}$ and $\left|\eta^{\gamma}\right|<1.5$. The estimated value of $A \times \epsilon_{\mathrm{MC}}$ for $M_{\chi}$ in the range $1-100 \mathrm{GeV}$ is between $30.5 \%-31.0 \%$ for vector and $29.2 \%-31.4 \%$ for axialvector couplings, respectively. The spectra for ADD MC events are generated using PYTHIA 8.145[23], requiring $p_{T}^{\gamma}>130 \mathrm{GeV}$, and scaled to NLO using a $K$ factor from Ref. [24]. The factor $A \times \epsilon_{\mathrm{MC}}$ for ADD is in the range of $26.5 \%-28.5 \%$ in the parameter space spanned by $n=3-6$ and $M_{D}=1-3 \mathrm{GeV}$.

Systematic uncertainties that contribute to the $A \times \epsilon_{\mathrm{MC}}$ calculation are from the choice of PDF [20,25,26], the selection of the primary vertex for the photon, modeling of pileup, and the energy calibration and resolution for photons [10], jets [27], and $\mathscr{E}_{T}$ [28]. The total systematic uncertainty on $A \times \epsilon_{\mathrm{MC}}$ is $+4.8 \%$ and $-4.9 \%$.

As mentioned above, $A \times \epsilon_{\mathrm{MC}}$ is multiplied by a SF to account for the difference in efficiency between data and MC predictions. The calculated SF of $0.90 \pm 0.11$ combines contributions from the trigger, photon reconstruction, consistency of cluster timing, and vetoes. The photon high level trigger is determined to be essentially $100 \%$ efficient for our selection criteria in data and in the MC simulation, but is assigned a $2 \%$ uncertainty due to small L1 trigger inefficiencies. Since the photon identification requirements have similar efficiencies for photons and electrons, the electron efficiency of $0.96 \pm 0.02$, as measured in $Z \rightarrow e e$ decays is used as the SF. Corrections for photon reconstruction are described in Ref. [22]. The photon clusters in MC simulations always have consistent timing among individual crystals, and the SF in data is found to be $0.983 \pm 0.009$ based on a sample of electron events. The track and jet-veto efficiency is studied in samples of $W \rightarrow e \nu$ data and MC, and confirmed with $Z \gamma \rightarrow e e \gamma$ data. Since the efficiencies measured in these samples agree within their uncertainties, the SF is set to unity and assigned a systematic uncertainty of \pm 0.10 . The SF for the 
TABLE I. (a) Observed (expected) 90\% C.L. upper limits on the DM production cross section $\sigma$, and $90 \%$ C.L. lower limits on the cutoff scale $\Lambda$ for vector and axial-vector operators as a function of the DM mass $M_{\chi}$. (b) Expected and observed lower limits on $M_{D}$ at $95 \%$ C.L., as a function of extra dimensions $n$, with $K$ factors (and without, i.e., $K=1$ ).

\begin{tabular}{|c|c|c|c|c|}
\hline & $0 \%$ C.L. Lin & its on DM & odel paramet & \\
\hline \multirow{2}{*}{$M_{\chi}[\mathrm{GeV}]$} & \multicolumn{2}{|c|}{ Vector } & \multicolumn{2}{|c|}{ Axial-Vector } \\
\hline & $\sigma[\mathrm{fb}]$ & $\Lambda[\mathrm{GeV}]$ & $\sigma[\mathrm{fb}]$ & $\Lambda[\mathrm{GeV}]$ \\
\hline 1 & $14.3(14.7)$ & $572(568)$ & $14.9(15.4)$ & $565(561)$ \\
\hline 10 & $14.3(14.7)$ & $571(567)$ & $14.1(14.5)$ & 573 (569) \\
\hline 100 & $15.4(15.3)$ & $558(558)$ & $13.9(14.3)$ & $554(550)$ \\
\hline 200 & $14.3(14.7)$ & $549(545)$ & $14.0(14.5)$ & $508(504)$ \\
\hline 500 & $13.6(14.0)$ & 442 (439) & $13.7(14.1)$ & $358(356)$ \\
\hline 1000 & $14.1(14.5)$ & $246(244)$ & $13.9(14.3)$ & $172(171)$ \\
\hline
\end{tabular}

(b) $95 \%$ C.L. Limits on ADD parameters.

\begin{tabular}{cccc} 
n & $K$ factors & $\begin{array}{c}\text { Expected } \\
M_{D}[\mathrm{TeV}]\end{array}$ & $\begin{array}{c}\text { Observed } \\
M_{D}[\mathrm{TeV}]\end{array}$ \\
\hline 3 & 1.5 & $1.70(1.53)$ & $1.73(1.55)$ \\
4 & 1.4 & $1.65(1.53)$ & $1.67(1.55)$ \\
5 & 1.3 & $1.63(1.54)$ & $1.64(1.56)$ \\
6 & 1.2 & $1.62(1.55)$ & $1.64(1.57)$ \\
\hline \hline
\end{tabular}

cosmic-ray muon veto is determined to be $0.95 \pm 0.01$ by comparing its efficiency in $\mathrm{MC}$ and data in a sample of $Z \rightarrow e e$ events.

Upper limits are placed on the DM production cross sections, as a function of $M_{\chi}$, assuming vector and axial-vector operators, summarized in Table I. These are converted into the corresponding lower limits on the cutoff scale $\Lambda$, also listed in Table I. The $\Lambda$ values are then translated into upper limits on the $\chi$-nucleon cross sections, calculated within the effective theory framework. These are displayed in Fig. 2 as a function of $M_{\chi}$ [2]. Superposed are the results from selected other experiments. Previously inaccessible $\chi$ masses below $\approx 3.5 \mathrm{GeV}$ are excluded for a $\chi$-nucleon cross section greater than $\approx 3 \mathrm{fb}$ at $90 \%$ C.L. For spin-dependent scattering, the upper limits surpass all previous constraints for the mass range of $1-100 \mathrm{GeV}$. The results presented are valid for mediator masses larger than the limits on $\Lambda$, assuming unity for the couplings $g_{\chi}$ and $g_{q}$. The specific case of light mediators is discussed in Refs. [3,30]. The assumptions on $\chi$ interactions made in calculating the limits vary with experiments. Further, in the case of direct and indirect searches, an astrophysical model must be assumed for the density and velocity distribution of DM.

A set of $95 \%$ C.L. upper limits are also placed on the ADD cross sections and translated into exclusions on the parameter space of the model. The upper limits are calculated using a C.L. method [31], with uncertainties parametrized by log-normal distributions in the fit to data. The limits on $M_{D}$, with and without $K$ factors, are summarized
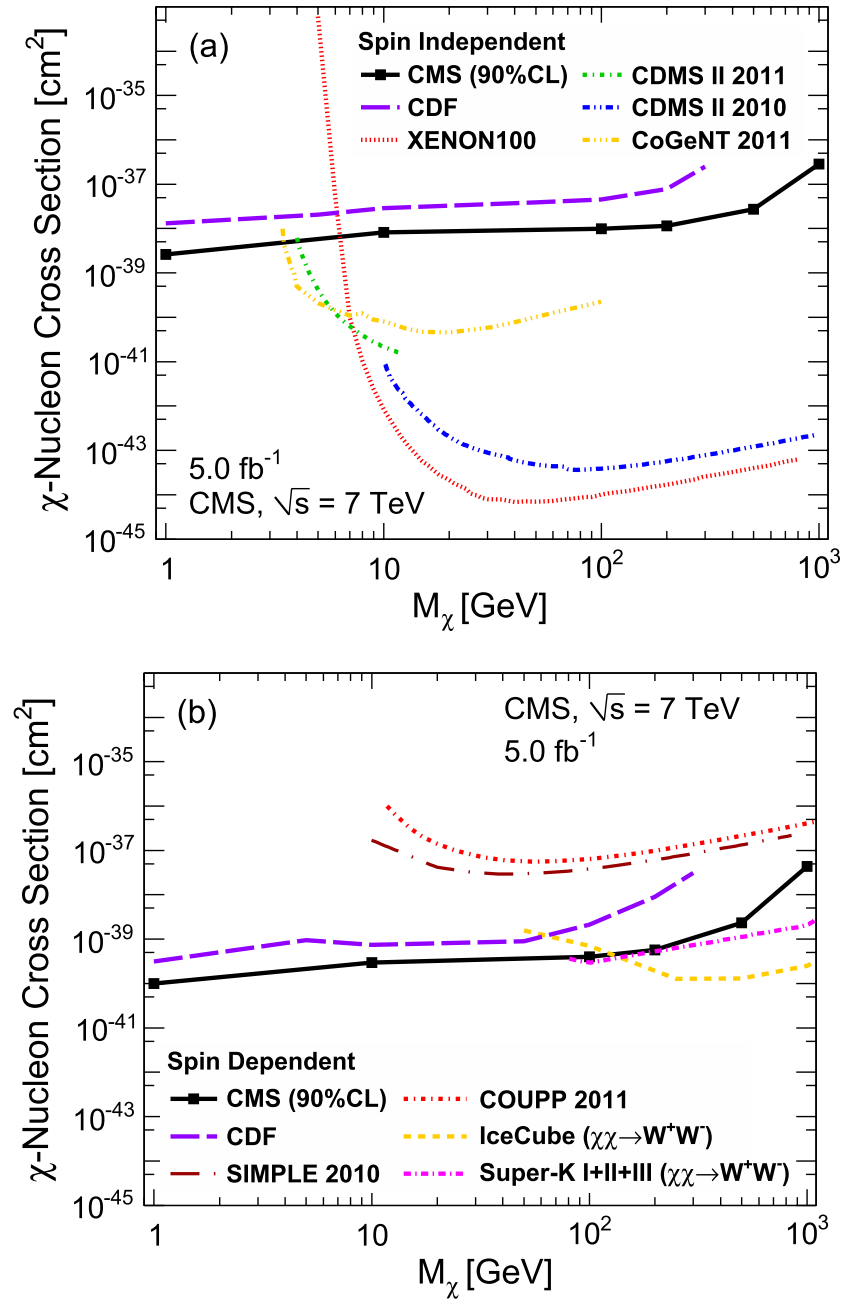

FIG. 2 (color online). The 90\% C.L. upper limits on the $\chi$-nucleon cross section as a function of $M_{\chi}$ for (a) spinindependent and (b) spin-dependent scattering. Also shown are the limits from selected experiments [29,35-42].

in Table I. Masses $M_{D}<1.64 \mathrm{TeV}$ are excluded at 95\% C.L. for $n=3$, assuming NLO cross sections. These limits, along with existing LO ADD limits from the Tevatron [32,33] and LEP [34], are shown in Fig. 3 as a function of $M_{D}$, for $n=4$ and $n=6$ extra dimensions. These results extend significantly the limits on the ADD model in the single-photon channel beyond previous measurements at the Tevatron and LEP experiments, and set limits of $M_{D}>1.64-1.73 \mathrm{TeV}$ for $n=3-6$ at 95\% C.L.

In summary, the agreement between single-photon production in $p p$ collisions at $7 \mathrm{TeV}$ and standard-model expectations was used to derive significant upper limits on the vector and axial-vector contributions to the $\chi$-nucleon scattering cross section. This search was complementary to searches for elastic $\chi$-nucleon scattering or $\chi \bar{\chi}$ annihilation. In addition, through greater sensitivity to the ADD model, the analysis attained the most stringent limits on an 

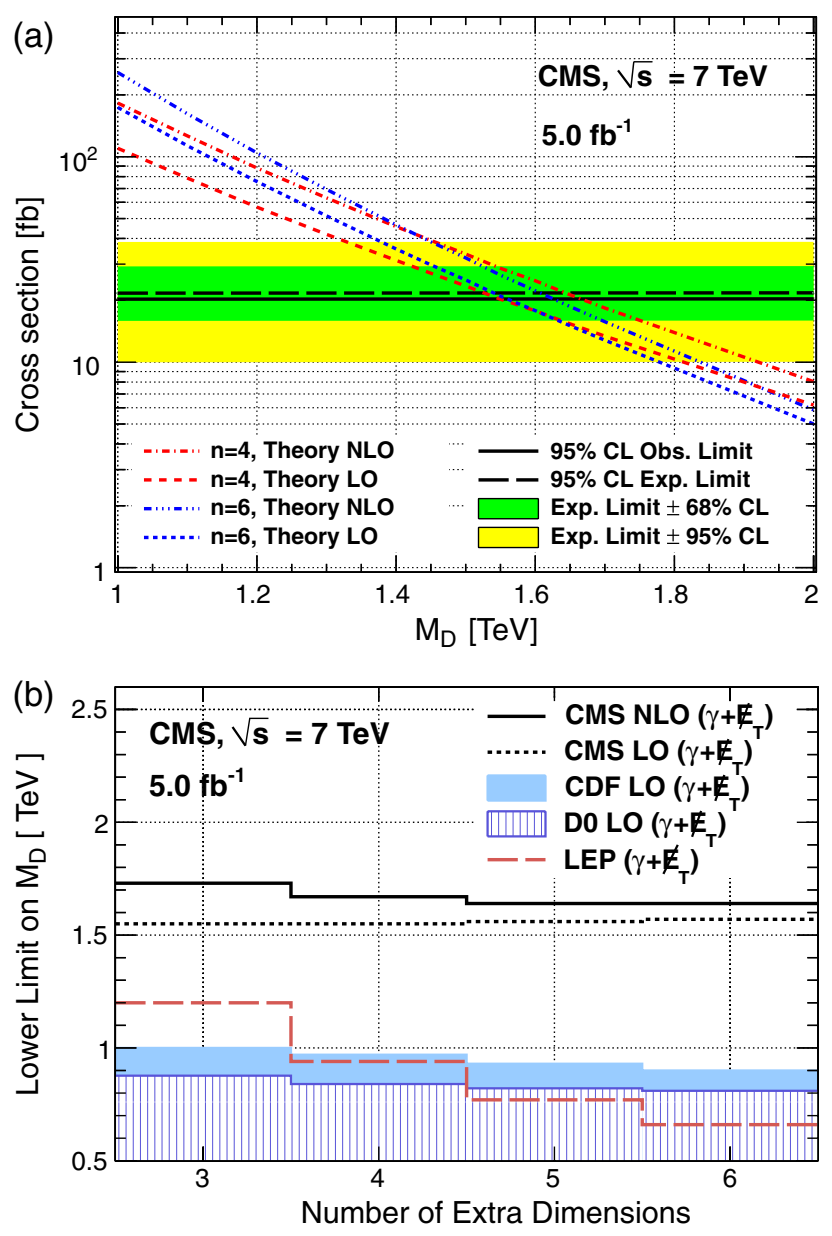

FIG. 3 (color online). (a) The 95\% C.L. upper limits on the LO and NLO ADD cross sections as a function of $M_{D}$ for $n=4$ and 6. (b) Limits on $M_{D}$ as a function of $n$, compared to LO results from similar searches at the Tevatron [32,33] and LEP [34].

effective extra-dimensional Planck scale obtained in the $\gamma+\not E_{T}$ production channel.

We thank R. Harnik, P. J. Fox, and J. Kopp for help in modeling dark-matter production. We congratulate our colleagues in the CERN accelerator departments for the excellent performance of the LHC machine. We thank the technical and administrative staff at CERN and other CMS institutes, and acknowledge support from FMSR (Austria); FNRS and FWO (Belgium); CNPq, CAPES, FAPERJ, and FAPESP (Brazil); MES (Bulgaria); CERN; CAS, MoST, and NSFC (China); COLCIENCIAS (Colombia); MSES (Croatia); RPF (Cyprus); MoER, SF0690030s09 and ERDF (Estonia); Academy of Finland, MEC, and HIP (Finland); CEA and CNRS/IN2P3 (France); BMBF, DFG, and HGF (Germany); GSRT (Greece); OTKA and NKTH (Hungary); DAE and DST (India); IPM (Iran); SFI (Ireland); INFN (Italy); NRF and WCU (Korea); LAS (Lithuania); CINVESTAV, CONACYT, SEP, and UASLP-FAI (Mexico); MSI (New Zealand); PAEC (Pakistan); MSHE and NSC (Poland); FCT (Portugal); JINR (Armenia, Belarus, Georgia, Ukraine, Uzbekistan);
MON, RosAtom, RAS and RFBR (Russia); MSTD (Serbia); MICINN and CPAN (Spain); Swiss Funding Agencies (Switzerland); NSC (Taipei); TUBITAK and TAEK (Turkey); STFC (U.K.); DOE and NSF (U.S.).

[1] R. Gaitskell, Annu. Rev. Nucl. Part. Sci. 54, 315 (2004).

[2] Y. Bai, P. J. Fox, and R. Harnik, J. High Energy Phys. 12 (2010) 048.

[3] P. J. Fox, R. Harnik, J. Kopp, and Y. Tsai, Phys. Rev. D 85, 056011 (2012).

[4] J. Goodman, M. Ibe, A. Rajaraman, W. Shepherd, T. M. P. Tait, and H.-B. Yu. Phys. Lett. B 695, 185 (2011).

[5] J. Goodman, M. Ibe, A. Rajaraman, W. Shepherd, T. M. P. Tait, and H.-B. Yu. Phys. Rev. D 82, (2010).

[6] N. Arkani-Hamed, S. Dimopoulos, and G. Dvali, Phys. Lett. B 429, 263 (1998).

[7] S. Chatrchyan et al. (CMS Collaboration), Phys. Rev. Lett. 107, 201804 (2011).

[8] G. Aad et al. (ATLAS Collaboration), Phys. Lett. B 705, 294 (2011).

[9] CMS Collaboration, J. Phys. G 34, 995 (2007).

[10] CMS Collaboration, CMS Physics Analysis Summary, Report No. CMS-PAS-EGM-10-006, 2011, http:// cdsweb.cern.ch/record/1324545? ln=en.

[11] CMS Collaboration, CMS Physics Analysis Summary, Report No. CMS-PAS-PFT-10-002, 2010, http://cdsweb .cern.ch/record/1279341? ln=en.

[12] CMS Collaboration, CMS Physics Analysis Summary, Report No. CMS-PAS-EGM-10-004, 2010, http:// cdsweb.cern.ch/record/1299116? ln=en.

[13] M. Cacciari, G. P. Salam, and G. Soyez, J. High Energy Phys. 04 (2008) 063.

[14] CMS Collaboration, CMS Physics Analysis Summary, Report No. CMS-NOTE-2010-012, 2010, http://cdsweb .cern.ch/record/1278160? $1 \mathrm{n}=\mathrm{en}$.

[15] S. Agostinelli et al. (GEANT4 Collaboration), Nucl. Instrum. Methods Phys. Res., Sect. A 506, 250 (2003).

[16] J. Allison et al., IEEE Trans. Nucl. Sci. 53, 270 (2006).

[17] J. Alwall, M. Herquet, F. Maltoni, O. Mattelaer, and T. Stelzer, J. High Energy Phys. 6 (2011) 128.

[18] J. Campbell, R. Ellis, and C. Williams, MCFM v6.1: A Monte Carlo for FeMtobarn processes at Hadron Colliders, http://mcfm.fnal.gov/mcfm.pdf (2011).

[19] T. Sjöstrand, S. Mrenna, and P.Z. Skands, J. High Energy Phys. 5 (2006) 026.

[20] J. Pumplin, D. Stump, J. Huston, H. Lai, P. Nadolsky, and W. Tung, J. High Energy Phys. 07 (2002) 012.

[21] U. Baur and E. Berger, Phys. Rev. D 47, 4889 (1993).

[22] S. Chatrchyan et al. (CMS Collaboration), Phys. Lett. B 701, 535 (2011).

[23] T. Sjöstrand, S. Mrenna, and P. Skands, Comput. Phys. Commun. 178, 852 (2008).

[24] X. Gao, C.S. Li, J. Gao, and J. Wang, Phys. Rev. D 81, 036008 (2010).

[25] M. Botje, J. Butterworth, A. Cooper-Sarkar, A. de Roeck, J. Feltesse, S. Forte, A. Glazov, J. Huston, R. McNulty, T. Sjöstrand, and R. Thorne, arXiv:1101.0538. 
[26] A. Martin, W. Stirling, R. Thorne, and G. Watt, Eur. Phys. J. C 63, 189 (2009).

[27] CMS Collaboration, JINST 6, P11002 (2011).

[28] CMS Collaboration, JINST 6, P09001 (2011).

[29] CDF Collaboration, Phys. Rev. Lett. 108, 211804 (2012).

[30] I. Shoemaker and L. Vecchi, arXiv:1112.5457.

[31] K. Nakamura et al. (Particle Data Group), J. Phys. G 37, 075021 (2010).

[32] T. Aaltonen et al. (CDF Collaboration), Phys. Rev. Lett. 101, 181602 (2008).

[33] V. M. Abazov et al. (D0 Collaboration), Phys. Rev. Lett. 101, 011601 (2008).

[34] J. Abdallah et al. (DELPHI Collaboration), Eur. Phys. J. C 38, 395 (2005).

[35] E. Aprile et al. (XENON100 Collaboration), Phys. Rev. Lett. 107, 131302 (2011).
[36] Z. Ahmed et al. (CDMS Collaboration), Phys. Rev. Lett. 106, 131302 (2011).

[37] CDMS II Collaboration, Science 327, 1619 (2010).

[38] C. E. Aalseth et al. (CoGeNT Collaboration), Phys. Rev. Lett. 106, 131301 (2011).

[39] M. Felizardo, T. Morlat, A.C. Fernandes, T. A. Girard, J. G. Marques, A. R. Ramos, M. Auguste, D. Boyer, A. Cavaillou, C. Sudre, J. Poupeney, R. F. Payne, H. S. Miley, and J. Puibasset (SIMPLE Collaboration), Phys. Rev. Lett. 105, 211301 (2010). A more recent update can be found in arXiv:1106.3014v3.

[40] E. Behnke et al., Phys. Rev. Lett. 106, 021303 (2011).

[41] R. Abbasi et al. (IceCube Collaboration), Phys. Rev. D 85, 042002 (2012).

[42] T. Tanaka et al., Astrophys. J. 742, 78 (2011).

S. Chatrchyan, ${ }^{1}$ V. Khachatryan, ${ }^{1}$ A. M. Sirunyan, ${ }^{1}$ A. Tumasyan, ${ }^{1}$ W. Adam, ${ }^{2}$ T. Bergauer, ${ }^{2}$ M. Dragicevic, ${ }^{2}$ J. Erö, ${ }^{2}$ C. Fabjan, ${ }^{2}$ M. Friedl, ${ }^{2}$ R. Frühwirth, ${ }^{2}$ V. M. Ghete, ${ }^{2}$ J. Hammer, ${ }^{2, b}$ N. Hörmann, ${ }^{2}$ J. Hrubec, ${ }^{2}$ M. Jeitler, ${ }^{2}$

W. Kiesenhofer, ${ }^{2}$ M. Krammer, ${ }^{2}$ D. Liko, ${ }^{2}$ I. Mikulec, ${ }^{2}$ M. Pernicka, ${ }^{2, a}$ B. Rahbaran, ${ }^{2}$ C. Rohringer, ${ }^{2}$ H. Rohringer, ${ }^{2}$ R. Schöfbeck, ${ }^{2}$ J. Strauss, ${ }^{2}$ A. Taurok, ${ }^{2}$ F. Teischinger, ${ }^{2}$ P. Wagner, ${ }^{2}$ W. Waltenberger, ${ }^{2}$ G. Walzel, ${ }^{2}$ E. Widl, ${ }^{2}$

C.-E. Wulz, ${ }^{2}$ V. Mossolov, ${ }^{3}$ N. Shumeiko, ${ }^{3}$ J. Suarez Gonzalez, ${ }^{3}$ S. Bansal, ${ }^{4}$ K. Cerny, ${ }^{4}$ T. Cornelis, ${ }^{4}$ E. A. De Wolf, ${ }^{4}$ X. Janssen, ${ }^{4}$ S. Luyckx,${ }^{4}$ T. Maes, ${ }^{4}$ L. Mucibello, ${ }^{4}$ S. Ochesanu, ${ }^{4}$ B. Roland, ${ }^{4}$ R. Rougny, ${ }^{4}$ M. Selvaggi, ${ }^{4}$

H. Van Haevermaet, ${ }^{4}$ P. Van Mechelen, ${ }^{4}$ N. Van Remortel, ${ }^{4}$ A. Van Spilbeeck, ${ }^{4}$ F. Blekman, ${ }^{5}$ S. Blyweert,${ }^{5}$

J. D'Hondt, ${ }^{5}$ R. Gonzalez Suarez, ${ }^{5}$ A. Kalogeropoulos, ${ }^{5}$ M. Maes, ${ }^{5}$ A. Olbrechts, ${ }^{5}$ W. Van Doninck, ${ }^{5}$

P. Van Mulders, ${ }^{5}$ G. P. Van Onsem, ${ }^{5}$ I. Villella ${ }^{5}$ O. Charaf,${ }^{6}$ B. Clerbaux, ${ }^{6}$ G. De Lentdecker, ${ }^{6}$ V. Dero, ${ }^{6}$ A. P. R. Gay, ${ }^{6}$

T. Hreus, ${ }^{6}$ A. Léonard, ${ }^{6}$ P. E. Marage, ${ }^{6}$ T. Reis, ${ }^{6}$ L. Thomas, ${ }^{6}$ C. Vander Velde, ${ }^{6}$ P. Vanlaer, ${ }^{6}$ V. Adler, ${ }^{7}$ K. Beernaert, ${ }^{7}$

A. Cimmino, ${ }^{7}$ S. Costantini, ${ }^{7}$ G. Garcia, ${ }^{7}$ M. Grunewald, ${ }^{7}$ B. Klein, ${ }^{7}$ J. Lellouch, ${ }^{7}$ A. Marinov, ${ }^{7}$ J. Mccartin, ${ }^{7}$

A. A. Ocampo Rios, ${ }^{7}$ D. Ryckbosch,${ }^{7}$ N. Strobbe, ${ }^{7}$ F. Thyssen, ${ }^{7}$ M. Tytgat,${ }^{7}$ L. Vanelderen, ${ }^{7}$ P. Verwilligen, ${ }^{7}$

S. Walsh, ${ }^{7}$ E. Yazgan, ${ }^{7}$ N. Zaganidis, ${ }^{7}$ S. Basegmez,${ }^{8}$ G. Bruno, ${ }^{8}$ L. Ceard, ${ }^{8}$ C. Delaere, ${ }^{8}$ T. du Pree, ${ }^{8}$ D. Favart, ${ }^{8}$

L. Forthomme, ${ }^{8}$ A. Giammanco, ${ }^{8, \mathrm{c}}$ J. Hollar, ${ }^{8}$ V. Lemaitre, ${ }^{8}$ J. Liao, ${ }^{8}$ O. Militaru, ${ }^{8}$ C. Nuttens, ${ }^{8}$ D. Pagano, ${ }^{8}$ A. Pin, ${ }^{8}$

K. Piotrzkowski, ${ }^{8}$ N. Schul,${ }^{8}$ N. Beliy, ${ }^{9}$ T. Caebergs, ${ }^{9}$ E. Daubie, ${ }^{9}$ G. H. Hammad,${ }^{9}$ G. A. Alves,${ }^{10}$

M. Correa Martins Junior, ${ }^{10}$ D. De Jesus Damiao, ${ }^{10}$ T. Martins, ${ }^{10}$ M. E. Pol,${ }^{10}$ M. H. G. Souza, ${ }^{10}$ W. L. Aldá Júnior, ${ }^{11}$

W. Carvalho, ${ }^{11}$ A. Custódio, ${ }^{11}$ E. M. Da Costa,${ }^{11}$ C. De Oliveira Martins, ${ }^{11}$ S. Fonseca De Souza,${ }^{11}$

D. Matos Figueiredo,${ }^{11}$ L. Mundim, ${ }^{11}$ H. Nogima,${ }^{11}$ V. Oguri, ${ }^{11}$ W. L. Prado Da Silva, ${ }^{11}$ A. Santoro, ${ }^{11}$

S. M. Silva Do Amaral, ${ }^{11}$ L. Soares Jorge, ${ }^{11}$ A. Sznajder, ${ }^{11}$ T. S. Anjos, ${ }^{12, \mathrm{~d}}$ C. A. Bernardes, ${ }^{12, \mathrm{~d}}$ F. A. Dias, ${ }^{12, \mathrm{e}}$

T. R. Fernandez Perez Tomei, ${ }^{12}$ E. M. Gregores, ${ }^{12, d}$ C. Lagana, ${ }^{12}$ F. Marinho, ${ }^{12}$ P. G. Mercadante, ${ }^{12, d}$ S. F. Novaes,${ }^{12}$

Sandra S. Padula, ${ }^{12}$ V. Genchev, ${ }^{13, b}$ P. Iaydjiev, ${ }^{13, b}$ S. Piperov, ${ }^{13}$ M. Rodozov, ${ }^{13}$ S. Stoykova, ${ }^{13}$ G. Sultanov, ${ }^{13}$

V. Tcholakov, ${ }^{13}$ R. Trayanov, ${ }^{13}$ M. Vutova, ${ }^{13}$ A. Dimitrov, ${ }^{14}$ R. Hadjiiska, ${ }^{14}$ V. Kozhuharov,${ }^{14}$ L. Litov, ${ }^{14}$ B. Pavlov,${ }^{14}$ P. Petkov, ${ }^{14}$ J. G. Bian, ${ }^{15}$ G. M. Chen, ${ }^{15}$ H. S. Chen, ${ }^{15}$ C. H. Jiang, ${ }^{15}$ D. Liang, ${ }^{15}$ S. Liang, ${ }^{15}$ X. Meng, ${ }^{15}$ J. Tao, ${ }^{15}$ J. Wang, ${ }^{15}$ J. Wang, ${ }^{15}$ X. Wang, ${ }^{15}$ Z. Wang, ${ }^{15}$ H. Xiao, ${ }^{15} \mathrm{M}$. Xu, ${ }^{15}$ J. Zang, ${ }^{15}$ Z. Zhang, ${ }^{15}$ C. Asawatangtrakuldee, ${ }^{16}$ Y. Ban, ${ }^{16}$ S. Guo, ${ }^{16}$ Y. Guo, ${ }^{16}$ W. Li, ${ }^{16}$ S. Liu, ${ }^{16}$ Y. Mao, ${ }^{16}$ S. J. Qian, ${ }^{16}$ H. Teng, ${ }^{16}$ S. Wang, ${ }^{16}$ B. Zhu, ${ }^{16}$ W. Zou, ${ }^{16}$ C. Avila, ${ }^{17}$ B. Gomez Moreno, ${ }^{17}$ A. F. Osorio Oliveros, ${ }^{17}$ J. C. Sanabria, ${ }^{17}$ N. Godinovic, ${ }^{18}$ D. Lelas,${ }^{18}$ R. Plestina,${ }^{18, f}$

D. Polic,${ }^{18}$ I. Puljak,${ }^{18, b}$ Z. Antunovic, ${ }^{19}$ M. Dzelalija, ${ }^{19}$ M. Kovac, ${ }^{19}$ V. Brigljevic, ${ }^{20}$ S. Duric, ${ }^{20}$ K. Kadija, ${ }^{20}$

J. Luetic, ${ }^{20}$ S. Morovic, ${ }^{20}$ A. Attikis, ${ }^{21}$ M. Galanti, ${ }^{21}$ G. Mavromanolakis, ${ }^{21}$ J. Mousa, ${ }^{21}$ C. Nicolaou, ${ }^{21}$ F. Ptochos, ${ }^{21}$

P. A. Razis, ${ }^{21}$ M. Finger, ${ }^{22}$ M. Finger, Jr., ${ }^{22}$ Y. Assran, ${ }^{23, g}$ S. Elgammal, ${ }^{23, h}$ A. Ellithi Kamel, ${ }^{23, i}$ S. Khalil, ${ }^{23, j}$

M. A. Mahmoud ${ }^{23, k}$ A. Radi, ${ }^{23, j}$ M. Kadastik, ${ }^{24}$ M. Müntel,${ }^{24}$ M. Raidal,${ }^{24}$ L. Rebane, ${ }^{24}$ A. Tiko, ${ }^{24}$ V. Azzolini, ${ }^{25}$ P. Eerola, ${ }^{25}$ G. Fedi, ${ }^{25}$ M. Voutilainen, ${ }^{25}$ J. Härkönen, ${ }^{26}$ A. Heikkinen, ${ }^{26}$ V. Karimäki, ${ }^{26}$ R. Kinnunen, ${ }^{26}$

M. J. Kortelainen, ${ }^{26}$ T. Lampén, ${ }^{26}$ K. Lassila-Perini, ${ }^{26}$ S. Lehti, ${ }^{26}$ T. Lindén, ${ }^{26}$ P. Luukka, ${ }^{26}$ T. Mäenpää, ${ }^{26}$

T. Peltola, ${ }^{26}$ E. Tuominen, ${ }^{26}$ J. Tuominiemi,${ }^{26}$ E. Tuovinen, ${ }^{26}$ D. Ungaro, ${ }^{26}$ L. Wendland, ${ }^{26} \mathrm{~K}$. Banzuzi, ${ }^{27}$

A. Korpela, ${ }^{27}$ T. Tuuva ${ }^{27}$ M. Besancon, ${ }^{28}$ S. Choudhury, ${ }^{28}$ M. Dejardin, ${ }^{28}$ D. Denegri, ${ }^{28}$ B. Fabbro, ${ }^{28}$ J. L. Faure, ${ }^{28}$ F. Ferri, ${ }^{28}$ S. Ganjour, ${ }^{28}$ A. Givernaud ${ }^{28}$ P. Gras, ${ }^{28}$ G. Hamel de Monchenault,${ }^{28}$ P. Jarry, ${ }^{28}$ E. Locci, ${ }^{28}$ J. Malcles ${ }^{28}$ L. Millischer, ${ }^{28}$ A. Nayak, ${ }^{28}$ J. Rander, ${ }^{28}$ A. Rosowsky, ${ }^{28}$ I. Shreyber, ${ }^{28}$ M. Titov,${ }^{28}$ S. Baffioni, ${ }^{29}$ F. Beaudette, ${ }^{29}$ 
L. Benhabib, ${ }^{29}$ L. Bianchini, ${ }^{29}$ M. Bluj, ${ }^{29,1}$ C. Broutin, ${ }^{29}$ P. Busson, ${ }^{29}$ C. Charlot, ${ }^{29}$ N. Daci, ${ }^{29}$ T. Dahms,${ }^{29}$

L. Dobrzynski, ${ }^{29}$ R. Granier de Cassagnac, ${ }^{29}$ M. Haguenauer, ${ }^{29}$ P. Miné, ${ }^{29}$ C. Mironov, ${ }^{29}$ C. Ochando,${ }^{29}$ P. Paganini, ${ }^{29}$ D. Sabes,${ }^{29}$ R. Salerno, ${ }^{29}$ Y. Sirois,${ }^{29}$ C. Veelken,${ }^{29}$ A. Zabi, ${ }^{29}$ J.-L. Agram, ${ }^{30, m}$ J. Andrea, ${ }^{30}$ D. Bloch, ${ }^{30}$ D. Bodin, ${ }^{30}$ J.-M. Brom,${ }^{30}$ M. Cardaci, ${ }^{30}$ E. C. Chabert,${ }^{30}$ C. Collard, ${ }^{30}$ E. Conte, ${ }^{30, m}$ F. Drouhin, ${ }^{30, m}$ C. Ferro, ${ }^{30}$ J.-C. Fontaine, ${ }^{30, m}$ D. Gelé, ${ }^{30}$ U. Goerlach, ${ }^{30}$ P. Juillot, ${ }^{30}$ M. Karim, ${ }^{30, m}$ A.-C. Le Bihan, ${ }^{30}$ P. Van Hove, ${ }^{30}$ F. Fassi, ${ }^{31}$ D. Mercier, ${ }^{31}$ S. Beauceron, ${ }^{32}$ N. Beaupere, ${ }^{32}$ O. Bondu, ${ }^{32}$ G. Boudoul,,${ }^{32}$ H. Brun, ${ }^{32}$ J. Chasserat, ${ }^{32}$ R. Chierici, ${ }^{32, b}$

D. Contardo, ${ }^{32}$ P. Depasse, ${ }^{32}$ H. El Mamouni, ${ }^{32}$ J. Fay, ${ }^{32}$ S. Gascon, ${ }^{32}$ M. Gouzevitch, ${ }^{32}$ B. Ille, ${ }^{32}$ T. Kurca, ${ }^{32}$

M. Lethuillier, ${ }^{32}$ L. Mirabito, ${ }^{32}$ S. Perries, ${ }^{32}$ V. Sordini, ${ }^{32}$ S. Tosi,${ }^{32}$ Y. Tschudi, ${ }^{32}$ P. Verdier, ${ }^{32}$ S. Viret,${ }^{32}$

Z. Tsamalaidze, ${ }^{33}$ G. Anagnostou, ${ }^{34}$ S. Beranek,${ }^{34}$ M. Edelhoff,${ }^{34}$ L. Feld,${ }^{34}$ N. Heracleous,${ }^{34}$ O. Hindrichs, ${ }^{34}$

R. Jussen,${ }^{34}$ K. Klein, ${ }^{34}$ J. Merz, ${ }^{34}$ A. Ostapchuk,${ }^{34}$ A. Perieanu, ${ }^{34}$ F. Raupach,${ }^{34}$ J. Sammet, ${ }^{34}$ S. Schael,${ }^{34}$ D. Sprenger ${ }^{34}$ H. Weber, ${ }^{34}$ B. Wittmer, ${ }^{34}$ V. Zhukov, ${ }^{34, n}$ M. Ata, ${ }^{35}$ J. Caudron, ${ }^{35}$ E. Dietz-Laursonn, ${ }^{35}$ D. Duchardt,${ }^{35}$ M. Erdmann, ${ }^{35}$ A. Güth,,${ }^{35}$ T. Hebbeker, ${ }^{35}$ C. Heidemann, ${ }^{35}$ K. Hoepfner,,${ }^{35}$ T. Klimkovich, ${ }^{35}$ D. Klingebiel, ${ }^{35}$ P. Kreuzer, ${ }^{35}$ D. Lanske, ${ }^{35, a}$ J. Lingemann, ${ }^{35}$ C. Magass, ${ }^{35}$ M. Merschmeyer, ${ }^{35}$ A. Meyer, ${ }^{35}$ M. Olschewski,${ }^{35}$ P. Papacz, ${ }^{35}$ H. Pieta, ${ }^{35}$ H. Reithler, ${ }^{35}$ S. A. Schmitz, ${ }^{35}$ L. Sonnenschein, ${ }^{35}$ J. Steggemann, ${ }^{35}$ D. Teyssier,${ }^{35}$

M. Weber, ${ }^{35}$ M. Bontenackels, ${ }^{36}$ V. Cherepanov, ${ }^{36}$ M. Davids, ${ }^{36}$ G. Flügge,${ }^{36} \mathrm{H}$. Geenen, ${ }^{36}$ M. Geisler, ${ }^{36}$ W. Haj Ahmad, ${ }^{36}$ F. Hoehle, ${ }^{36}$ B. Kargoll, ${ }^{36}$ T. Kress, ${ }^{36}$ Y. Kuessel, ${ }^{36}$ A. Linn, ${ }^{36}$ A. Nowack, ${ }^{36}$ L. Perchalla, ${ }^{36}$ O. Pooth, ${ }^{36}$ J. Rennefeld, ${ }^{36}$ P. Sauerland, ${ }^{36}$ A. Stahl,${ }^{36}$ M. Aldaya Martin, ${ }^{37}$ J. Behr, ${ }^{37}$ W. Behrenhoff,,${ }^{37}$ U. Behrens, ${ }^{37}$ M. Bergholz, ${ }^{37, o}$ A. Bethani, ${ }^{37}$ K. Borras, ${ }^{37}$ A. Burgmeier, ${ }^{37}$ A. Cakir, ${ }^{37}$ L. Calligaris, ${ }^{37}$ A. Campbell,,${ }^{37}$ E. Castro, ${ }^{37}$ F. Costanza, ${ }^{37}$ D. Dammann, ${ }^{37}$ G. Eckerlin, ${ }^{37}$ D. Eckstein, ${ }^{37}$ D. Fischer,${ }^{37}$ G. Flucke, ${ }^{37}$ A. Geiser, ${ }^{37}$ I. Glushkov, ${ }^{37}$

S. Habib,${ }^{37}$ J. Hauk,${ }^{37}$ H. Jung, ${ }^{37, b}$ M. Kasemann, ${ }^{37}$ P. Katsas, ${ }^{37}$ C. Kleinwort, ${ }^{37}$ H. Kluge, ${ }^{37}$ A. Knutsson, ${ }^{37}$ M. Krämer, ${ }^{37}$ D. Krücker, ${ }^{37}$ E. Kuznetsova, ${ }^{37}$ W. Lange, ${ }^{37}$ W. Lohmann, ${ }^{37, o}$ B. Lutz, ${ }^{37}$ R. Mankel, ${ }^{37}$ I. Marfin,${ }^{37}$ M. Marienfeld, ${ }^{37}$ I.-A. Melzer-Pellmann, ${ }^{37}$ A. B. Meyer,${ }^{37}$ J. Mnich,${ }^{37}$ A. Mussgiller, ${ }^{37}$ S. Naumann-Emme, ${ }^{37}$ J. Olzem,,${ }^{37}$ H. Perrey, ${ }^{37}$ A. Petrukhin, ${ }^{37}$ D. Pitzl, ${ }^{37}$ A. Raspereza,${ }^{37}$ P. M. Ribeiro Cipriano, ${ }^{37}$ C. Riedl, ${ }^{37}$ M. Rosin, ${ }^{37}$ J. Salfeld-Nebgen, ${ }^{37}$ R. Schmidt, ${ }^{37,0}$ T. Schoerner-Sadenius, ${ }^{37}$ N. Sen,${ }^{37}$ A. Spiridonov, ${ }^{37}$ M. Stein, ${ }^{37}$ R. Walsh,${ }^{37}$ C. Wissing,${ }^{37}$ C. Autermann, ${ }^{38}$ V. Blobel,${ }^{38}$ S. Bobrovskyi,${ }^{38}$ J. Draeger, ${ }^{38}$ H. Enderle, ${ }^{38}$ J. Erfle, ${ }^{38}$ U. Gebbert,${ }^{38}$ M. Görner, ${ }^{38}$ T. Hermanns, ${ }^{38}$ R. S. Höing, ${ }^{38}$ K. Kaschube, ${ }^{38}$ G. Kaussen, ${ }^{38}$ H. Kirschenmann, ${ }^{38}$ R. Klanner ${ }^{38}$

J. Lange, ${ }^{38}$ B. Mura, ${ }^{38}$ F. Nowak, ${ }^{38}$ N. Pietsch, ${ }^{38}$ D. Rathjens, ${ }^{38}$ C. Sander,${ }^{38}$ H. Schettler ${ }^{38}$ P. Schleper, ${ }^{38}$ E. Schlieckau, ${ }^{38}$ A. Schmidt, ${ }^{38}$ M. Schröder, ${ }^{38}$ T. Schum, ${ }^{38}$ M. Seidel,${ }^{38}$ H. Stadie, ${ }^{38}$ G. Steinbrück, ${ }^{38}$ J. Thomsen, ${ }^{38}$ C. Barth, ${ }^{39}$ J. Berger, ${ }^{39}$ T. Chwalek, ${ }^{39}$ W. De Boer, ${ }^{39}$ A. Dierlamm, ${ }^{39}$ M. Feindt, ${ }^{39}$ M. Guthoff, ${ }^{39, b}$ C. Hackstein, ${ }^{39}$

F. Hartmann, ${ }^{39}$ M. Heinrich, ${ }^{39}$ H. Held,${ }^{39}$ K. H. Hoffmann, ${ }^{39}$ S. Honc, ${ }^{39}$ U. Husemann, ${ }^{39}$ I. Katkov, ${ }^{39, n}$

J. R. Komaragiri, ${ }^{39}$ D. Martschei, ${ }^{39}$ S. Mueller, ${ }^{39}$ Th. Müller, ${ }^{39}$ M. Niegel, ${ }^{39}$ A. Nürnberg, ${ }^{39}$ O. Oberst, ${ }^{39}$ A. Oehler,${ }^{39}$

J. Ott, ${ }^{39}$ T. Peiffer, ${ }^{39}$ G. Quast, ${ }^{39}$ K. Rabbertz, ${ }^{39}$ F. Ratnikov, ${ }^{39}$ N. Ratnikova, ${ }^{39}$ S. Röcker, ${ }^{39}$ C. Saout,${ }^{39}$

A. Scheurer, ${ }^{39}$ F.-P. Schilling, ${ }^{39}$ M. Schmanau, ${ }^{39}$ G. Schott, ${ }^{39}$ H. J. Simonis, ${ }^{39}$ F. M. Stober,,${ }^{39}$ D. Troendle, ${ }^{39}$

R. Ulrich, ${ }^{39}$ J. Wagner-Kuhr, ${ }^{39}$ T. Weiler,${ }^{39}$ M. Zeise, ${ }^{39}$ E. B. Ziebarth, ${ }^{39}$ G. Daskalakis, ${ }^{40}$ T. Geralis, ${ }^{40}$

S. Kesisoglou, ${ }^{40}$ A. Kyriakis, ${ }^{40}$ D. Loukas, ${ }^{40}$ I. Manolakos, ${ }^{40}$ A. Markou, ${ }^{40}$ C. Markou, ${ }^{40}$ C. Mavrommatis, ${ }^{40}$ E. Ntomari ${ }^{40}$ L. Gouskos, ${ }^{41}$ T. J. Mertzimekis, ${ }^{41}$ A. Panagiotou, ${ }^{41}$ N. Saoulidou, ${ }^{41}$ I. Evangelou, ${ }^{42}$ C. Foudas, ${ }^{42, b}$ P. Kokkas, ${ }^{42}$ N. Manthos, ${ }^{42}$ I. Papadopoulos, ${ }^{42}$ V. Patras,${ }^{42}$ G. Bencze,${ }^{43}$ C. Hajdu, ${ }^{43, b}$ P. Hidas, ${ }^{43}$ D. Horvath, ${ }^{43, p}$ K. Krajczar, ${ }^{43, q}$ B. Radics, ${ }^{43}$ F. Sikler, ${ }^{43, b}$ V. Veszpremi, ${ }^{43}$ G. Vesztergombi, ${ }^{43, q}$ N. Beni, ${ }^{44}$ S. Czellar, ${ }^{44}$ J. Molnar, ${ }^{44}$ J. Palinkas, ${ }^{44}$ Z. Szillasi, ${ }^{44}$ J. Karancsi, ${ }^{45}$ P. Raics, ${ }^{45}$ Z. L. Trocsanyi ${ }^{45}$ B. Ujvari, ${ }^{45}$ S. B. Beri, ${ }^{46}$ V. Bhatnagar ${ }^{46}$ N. Dhingra, ${ }^{46}$ R. Gupta, ${ }^{46}$ M. Jindal, ${ }^{46}$ M. Kaur, ${ }^{46}$ J. M. Kohli, ${ }^{46}$ M. Z. Mehta, ${ }^{46}$ N. Nishu, ${ }^{46}$ L. K. Saini, ${ }^{46}$ A. Sharma, ${ }^{46}$ J. Singh, ${ }^{46}$ S. P. Singh,${ }^{46}$ S. Ahuja, ${ }^{47}$ B. C. Choudhary,${ }^{47}$ A. Kumar, ${ }^{47}$ A. Kumar, ${ }^{47}$ S. Malhotra ${ }^{47}$ M. Naimuddin, ${ }^{47}$ K. Ranjan, ${ }^{47}$ V. Sharma,${ }^{47}$ R. K. Shivpuri,${ }^{47}$ S. Banerjee, ${ }^{48}$ S. Bhattacharya,${ }^{48}$ S. Dutta ${ }^{48}$ B. Gomber ${ }^{48}$ Sa. Jain, ${ }^{48}$ Sh. Jain, ${ }^{48}$ R. Khurana, ${ }^{48}$ S. Sarkar, ${ }^{48}$ A. Abdulsalam, ${ }^{49}$ R. K. Choudhury, ${ }^{49}$ D. Dutta, ${ }^{49}$ S. Kailas, ${ }^{49}$ V. Kumar, ${ }^{49}$ A. K. Mohanty, ${ }^{49, b}$ L. M. Pant,${ }^{49}$ P. Shukla, ${ }^{49}$ T. Aziz,${ }^{50}$ S. Ganguly, ${ }^{50}$ M. Guchait,${ }^{50, r}$ A. Gurtu, ${ }^{50}$ M. Maity, ${ }^{50, \mathrm{~s}}$ G. Majumder, ${ }^{50}$ K. Mazumdar, ${ }^{50}$ G. B. Mohanty, ${ }^{50}$ B. Parida,${ }^{50}$ K. Sudhakar, ${ }^{50}$

N. Wickramage ${ }^{50}$ S. Banerjee, ${ }^{51}$ S. Dugad,${ }^{51}$ H. Arfaei,${ }^{52}$ H. Bakhshiansohi,${ }^{52, t}$ S. M. Etesami, ${ }^{52, \mathrm{u}}$ A. Fahim,${ }^{52, t}$ M. Hashemi, ${ }^{52}$ H. Hesari, ${ }^{52}$ A. Jafari, ${ }^{52, t}$ M. Khakzad, ${ }^{52}$ A. Mohammadi, ${ }^{52, v}$ M. Mohammadi Najafabadi, ${ }^{52}$ S. Paktinat Mehdiabadi, ${ }^{52}$ B. Safarzadeh,${ }^{52, \mathrm{w}}$ M. Zeinali, ${ }^{52, \mathrm{u}}$ M. Abbrescia ${ }^{53 \mathrm{a}, 53 \mathrm{~b}}$ L. Barbone,${ }^{53 \mathrm{a}, 53 \mathrm{~b}}$

C. Calabria, ${ }^{53 a, 53 b, b}$ S. S. Chhibra, ${ }^{53 a, 53 b}$ A. Colaleo, ${ }^{53 a}$ D. Creanza, ${ }^{53 a, 53 c}$ N. De Filippis, ${ }^{53 a, 53 c, b}$ M. De Palma ${ }^{53 a, 53 b}$ L. Fiore, ${ }^{53 a}$ G. Iaselli, ${ }^{53 a, 53 c}$ L. Lusito, ${ }^{53 a, 53 b}$ G. Maggi, ${ }^{53 a, 53 c}$ M. Maggi, ${ }^{53 a}$ B. Marangelli, ${ }^{53 a, 53 b}$ S. My, ${ }^{53 a, 53 c}$ S. Nuzzo, ${ }^{53 a, 53 b}$ N. Pacifico, ${ }^{53 a, 53 b}$ A. Pompili, ${ }^{53 a, 53 b}$ G. Pugliese, ${ }^{53 a, 53 c}$ G. Selvaggi, ${ }^{53 a, 53 b}$ L. Silvestris, ${ }^{53 a}$ 
G. Singh, ${ }^{53 a, 53 b}$ G. Zito, ${ }^{53 a}$ G. Abbiendi, ${ }^{54 a}$ A. C. Benvenuti, ${ }^{54 a}$ D. Bonacorsi, ${ }^{54 a, 54 b}$ S. Braibant-Giacomelli, ${ }^{54 a, 54 b}$ L. Brigliadori, ${ }^{54 a, 54 b}$ P. Capiluppi, ${ }^{54 a, 54 b}$ A. Castro, ${ }^{54 a, 54 b}$ F. R. Cavallo, ${ }^{54 a}$ M. Cuffiani, ${ }^{54 a, 54 b}$ G. M. Dallavalle, ${ }^{54 a}$ F. Fabbri, ${ }^{54 a}$ A. Fanfani, ${ }^{54 a, 54 b}$ D. Fasanella, ${ }^{54 a, 54 b, b}$ P. Giacomelli, ${ }^{54 a}$ C. Grandi ${ }^{54 a}$ L. Guiducci, ${ }^{54 a}$ S. Marcellini, ${ }^{54 a}$ G. Masetti, ${ }^{54 a}$ M. Meneghelli, ${ }^{54 a, 54 b, b}$ A. Montanari, ${ }^{54 a}$ F. L. Navarria, ${ }^{54 a, 54 b}$ F. Odorici, ${ }^{54 a}$ A. Perrotta, ${ }^{54 a}$ F. Primavera, ${ }^{54 a, 54 b}$ A. M. Rossi, ${ }^{54 a, 54 b}$ T. Rovelli, ${ }^{54 a, 54 b}$ G. Siroli, ${ }^{54 a, 54 b}$ R. Travaglini,,${ }^{54,54 b}$ S. Albergo, ${ }^{55 a, 55 b}$ G. Cappello, ${ }^{55 a, 55 b}$ M. Chiorboli, ${ }^{55 a, 55 b}$ S. Costa, ${ }^{55 a, 55 b}$ R. Potenza, ${ }^{55 a, 55 b}$ A. Tricomi, ${ }^{55 a, 55 b}$ C. Tuve, ${ }^{55 a, 55 b}$ G. Barbagli, ${ }^{56 a}$ V. Ciulli, ${ }^{56,56 b}$ C. Civinini, ${ }^{56 a}$ R. D'Alessandro, ${ }^{56 a, 56 b}$ E. Focardi, ${ }^{56 a, 56 b}$ S. Frosali, ${ }^{56 a, 56 b}$ E. Gallo, ${ }^{56 a}$ S. Gonzi, ${ }^{56 a, 56 b}$ M. Meschini, ${ }^{56 a}$ S. Paoletti, ${ }^{56 a}$ G. Sguazzoni, ${ }^{56 a}$ A. Tropiano, ${ }^{56 a, b}$ L. Benussi, ${ }^{57}$ S. Bianco, ${ }^{57}$ S. Colafranceschi, ${ }^{57, x}$ F. Fabbri, ${ }^{57}$ D. Piccolo, ${ }^{57}$ P. Fabbricatore, ${ }^{58}$ R. Musenich,${ }^{58}$ A. Benaglia, ${ }^{59 a, 59 b, b}$ F. De Guio, ${ }^{59 a, 59 b}$ L. Di Matteo, ${ }^{59 a, 59 b, b}$ S. Fiorendi, ${ }^{59 a, 59 b}$ S. Gennai, ${ }^{59 a, b}$ A. Ghezzi, ${ }^{59 a, 59 b}$ S. Malvezzi, ${ }^{59 a}$ R. A. Manzoni, ${ }^{59 a, 59 b}$ A. Martelli ${ }^{59 a, 59 b}$ A. Massironi, ${ }^{59 a, 59 b, b}$ D. Menasce, ${ }^{59 a}$ L. Moroni, ${ }^{59 a}$ M. Paganoni, ${ }^{59 a, 59 b}$ D. Pedrini, ${ }^{59 a}$ S. Ragazzi, ${ }^{59 a, 59 b}$ N. Redaelli, ${ }^{59 a}$ S. Sala, ${ }^{59 a}$ T. Tabarelli de Fatis, ${ }^{59 a, 59 b}$ S. Buontempo, ${ }^{60 a}$ C. A. Carrillo Montoya ${ }^{60 a, b}$ N. Cavallo, ${ }^{60 a, y}$ A. De Cosa,${ }^{60 a, 60 b}$ O. Dogangun, ${ }^{60 a, 60 b}$ F. Fabozzi, ${ }^{60 a, y}$ A. O. M. Iorio, ${ }^{60 a, b}$ L. Lista ${ }^{60 a}$ S. Meola ${ }^{60 a, z}$ M. Merola,${ }^{60 a, 60 b}$ P. Paolucci, ${ }^{60 a}$ P. Azzi ${ }^{61 a}$ N. Bacchetta, ${ }^{61 a, b}$ D. Bisello, ${ }^{61 a, 61 b}$ A. Branca, ${ }^{61 \mathrm{a}, \mathrm{b}}$ R. Carlin, ${ }^{61 \mathrm{a}, 61 \mathrm{~b}}$ P. Checchia, ${ }^{61 \mathrm{a}}$ T. Dorigo, ${ }^{61 \mathrm{a}}$ U. Dosselli, ${ }^{61 \mathrm{a}} \mathrm{F}$. Gasparini, ${ }^{61 \mathrm{a}, 61 \mathrm{~b}}$ A. Gozzelino, ${ }^{61 \mathrm{a}}$ K. Kanishchev, ${ }^{61 \mathrm{a}, 61 \mathrm{c}}$ S. Lacaprara, ${ }^{61 \mathrm{a}}$ I. Lazzizzera, ${ }^{61 \mathrm{a}, 61 \mathrm{c}}$ M. Margoni, ${ }^{61 \mathrm{a}, 61 \mathrm{~b}}$

A. T. Meneguzzo, ${ }^{61 \mathrm{a}, 61 \mathrm{~b}}$ M. Nespolo, ${ }^{61 \mathrm{a}, \mathrm{b}}$ L. Perrozzi, ${ }^{61 \mathrm{a}}$ N. Pozzobon, ${ }^{61 \mathrm{a}, 61 \mathrm{~b}}$ P. Ronchese,${ }^{61 \mathrm{a}, 61 \mathrm{~b}}$ F. Simonetto, ${ }^{61 \mathrm{a}, 61 \mathrm{~b}}$ E. Torassa, ${ }^{61 a}$ M. Tosi, ${ }^{61 a, 61 b, b}$ S. Vanini, ${ }^{61 a, 61 b}$ P. Zotto, ${ }^{61 a, 61 b}$ G. Zumerle, ${ }^{61 a, 61 b}$ M. Gabusi, ${ }^{62 a, 62 b}$ S. P. Ratti, ${ }^{62 a, 62 b}$ C. Riccardi, ${ }^{62 a, 62 b}$ P. Torre, ${ }^{62 a, 62 b}$ P. Vitulo, ${ }^{62 a, 62 b}$ G. M. Bilei, ${ }^{63 a}$ L. Fanò, ${ }^{63 a, 63 b}$ P. Lariccia, ${ }^{63 a, 63 b}$

A. Lucaroni, ${ }^{63 a, 63 b, b}$ G. Mantovani,${ }^{63 a, 63 b}$ M. Menichelli, ${ }^{63 a}$ A. Nappi,${ }^{63 a, 63 b}$ F. Romeo, ${ }^{63 a, 63 b}$ A. Saha ${ }^{63 a}$ A. Santocchia ${ }^{63 a, 63 b}$ S. Taroni,${ }^{63 a, 63 b, b}$ P. Azzurri, ${ }^{64 a, 64 c}$ G. Bagliesi, ${ }^{64 a}$ T. Boccali, ${ }^{64 a}$ G. Broccolo, ${ }^{64 a, 64 c}$ R. Castaldi, ${ }^{64 a}$ R. T. D’Agnolo, ${ }^{64 a, 64 c}$ R. Dell'Orso, ${ }^{64 a}$ F. Fiori, ${ }^{64 a, 64 b, b}$ L. Foà, ${ }^{64 a, 64 c}$ A. Giassi, ${ }^{64 a}$ A. Kraan, ${ }^{64 a}$ F. Ligabue, ${ }^{64 a, 64 c}$ T. Lomtadze,${ }^{64 a}$ L. Martini,${ }^{64 a, a a}$ A. Messineo, ${ }^{64 a, 64 b}$ F. Palla, ${ }^{64 a}$ F. Palmonari, ${ }^{64 a}$ A. Rizzi, ${ }^{64 a, 64 b}$ A. T. Serban, ${ }^{64 a, b b}$ P. Spagnolo, ${ }^{64 a}$ P. Squillacioti, ${ }^{64 a, b}$ R. Tenchini, ${ }^{64 a}$ G. Tonelli, ${ }^{64 a, 64 b, b}$ A. Venturi, ${ }^{64 a, b}$ P. G. Verdini, ${ }^{64 a}$ L. Barone, ${ }^{65 a, 65 b}$ F. Cavallari, ${ }^{65 a}$ D. Del Re,${ }^{65 a, 65 b, b}$ M. Diemoz, ${ }^{65 a}$ C. Fanelli, ${ }^{65 a, 65 b}$ M. Grassi, ${ }^{65 a, b}$ E. Longo, ${ }^{65 a, 65 b}$ P. Meridiani, ${ }^{65 a, b}$ F. Micheli, ${ }^{65 a, 65 b}$ S. Nourbakhsh, ${ }^{65 a}$ G. Organtini, ${ }^{65 a, 65 b}$ F. Pandolfi, ${ }^{65 a, 65 b}$ R. Paramatti, ${ }^{65 a}$ S. Rahatlou, ${ }^{65 a, 65 b}$ M. Sigamani, ${ }^{65 a}$ L. Soffi, ${ }^{65 a, 65 b}$ N. Amapane, ${ }^{66 a, 66 b}$ R. Arcidiacono, ${ }^{66 a, 66 c}$ S. Argiro, ${ }^{66 a, 66 b}$ M. Arneodo, ${ }^{66 a, 66 c}$ C. Biino, ${ }^{66 a}$ C. Botta, ${ }^{66 a, 66 b}$ N. Cartiglia, ${ }^{66 a}$ R. Castello, ${ }^{66 a, 66 b}$ M. Costa, ${ }^{66 a, 66 b}$ N. Demaria ${ }^{66 \mathrm{a}}$ A. Graziano, ${ }^{66 \mathrm{a}, 66 \mathrm{~b}}$ C. Mariotti, ${ }^{66 \mathrm{a}, \mathrm{b}}$ S. Maselli, ${ }^{66 \mathrm{a}}$ E. Migliore ${ }^{66 \mathrm{a}, 66 \mathrm{~b}}$ V. Monaco, ${ }^{66 \mathrm{a}, 66 \mathrm{~b}}$ M. Musich, ${ }^{66 a, b}$ M. M. Obertino, ${ }^{66 a, 66 c}$ N. Pastrone, ${ }^{66 \mathrm{a}}$ M. Pelliccioni, ${ }^{66 \mathrm{a}}$ A. Potenza, ${ }^{66 a, 66 \mathrm{~b}}$ A. Romero, ${ }^{66 \mathrm{a}, 66 \mathrm{~b}}$ M. Ruspa,${ }^{66 a, 66 c}$ R. Sacchi, ${ }^{66 a, 66 b}$ A. Solano, ${ }^{66 a, 66 b}$ A. Staiano, ${ }^{66 a}$ A. Vilela Pereira, ${ }^{66 a}$ L. Visca, ${ }^{66 a, 66 b}$ S. Belforte, ${ }^{67 a}$ F. Cossutti, ${ }^{67 a}$ G. Della Ricca, ${ }^{67 a, 67 b}$ B. Gobbo, ${ }^{67 a}$ M. Marone, ${ }^{67 a, 67 b, b}$ D. Montanino, ${ }^{67 a, 67 b, b}$ A. Penzo, ${ }^{67 a}$ A. Schizzi, ${ }^{67 a, 67 b}$ S. G. Heo, ${ }^{68}$ T. Y. Kim, ${ }^{68}$ S. K. Nam ${ }^{68}$ S. Chang, ${ }^{69}$ J. Chung, ${ }^{69}$ D. H. Kim, ${ }^{69}$ G. N. Kim, ${ }^{69}$ D. J. Kong, ${ }^{69}$ H. Park, ${ }^{69}$ S. R. Ro, ${ }^{69}$ D. C. Son, ${ }^{69}$ T. Son,${ }^{69}$ J. Y. Kim, ${ }^{70}$ Zero J. Kim, ${ }^{70}$ S. Song,${ }^{70}$ H. Y. Jo, ${ }^{71}$ S. Choi, ${ }^{72}$ D. Gyun, ${ }^{72}$ B. Hong,${ }^{72}$ M. Jo, ${ }^{72}$ H. Kim, ${ }^{72}$ T. J. Kim, ${ }^{72}$ K. S. Lee, ${ }^{72}$ D. H. Moon ${ }^{72}$ S. K. Park,${ }^{72}$ E. Seo,${ }^{72}$ M. Choi ${ }^{73}$ S. Kang, ${ }^{73}$ H. Kim, ${ }^{73}$ J. H. Kim, ${ }^{73}$ C. Park ${ }^{73}$ I. C. Park, ${ }^{73}$ S. Park, ${ }^{73}$ G. Ryu,${ }^{73}$ Y. Cho, ${ }^{74}$ Y. Choi ${ }^{74}$ Y. K. Choi,${ }^{74}$ J. Goh, ${ }^{74}$ M. S. Kim,${ }^{74}$ E. Kwon,${ }^{74}$ B. Lee,${ }^{74}$ J. Lee, ${ }^{74}$ S. Lee,${ }^{74}$ H. Seo, ${ }^{74}$ I. Yu, ${ }^{74}$ M. J. Bilinskas, ${ }^{75}$ I. Grigelionis,${ }^{75}$ M. Janulis, ${ }^{75}$ A. Juodagalvis, ${ }^{75}$ H. Castilla-Valdez ${ }^{76}$ E. De La Cruz-Burelo, ${ }^{76}$ I. Heredia-de La Cruz,${ }^{76}$

R. Lopez-Fernandez, ${ }^{76}$ R. Magaña Villalba, ${ }^{76}$ J. Martínez-Ortega, ${ }^{76}$ A. Sánchez-Hernández, ${ }^{76}$

L. M. Villasenor-Cendejas, ${ }^{76}$ S. Carrillo Moreno, ${ }^{77}$ F. Vazquez Valencia, ${ }^{77}$ H. A. Salazar Ibarguen, ${ }^{78}$

E. Casimiro Linares, ${ }^{79}$ A. Morelos Pineda, ${ }^{79}$ M. A. Reyes-Santos,${ }^{79}$ D. Krofcheck, ${ }^{80}$ A. J. Bell, ${ }^{81}$ P. H. Butler, ${ }^{81}$ R. Doesburg, ${ }^{81}$ S. Reucroft, ${ }^{81}$ H. Silverwood ${ }^{81}$ M. Ahmad, ${ }^{82}$ M. I. Asghar,${ }^{82}$ H. R. Hoorani, ${ }^{82}$ S. Khalid ${ }^{82}$ W. A. Khan, ${ }^{82}$ T. Khurshid, ${ }^{82}$ S. Qazi, ${ }^{82}$ M. A. Shah, ${ }^{82}$ M. Shoaib, ${ }^{82}$ G. Brona, ${ }^{83}$ K. Bunkowski, ${ }^{83}$ M. Cwiok, ${ }^{83}$ W. Dominik ${ }^{83}$ K. Doroba, ${ }^{83}$ A. Kalinowski, ${ }^{83}$ M. Konecki, ${ }^{83}$ J. Krolikowski, ${ }^{83}$ H. Bialkowska, ${ }^{84}$ B. Boimska, ${ }^{84}$ T. Frueboes, ${ }^{84}$ R. Gokieli, ${ }^{84}$ M. Górski, ${ }^{84}$ M. Kazana, ${ }^{84}$ K. Nawrocki, ${ }^{84}$ K. Romanowska-Rybinska, ${ }^{84}$ M. Szleper,${ }^{84}$ G. Wrochna, ${ }^{84}$ P. Zalewski, ${ }^{84}$ N. Almeida ${ }^{85}$ P. Bargassa ${ }^{85}$ A. David, ${ }^{85}$ P. Faccioli ${ }^{85}$ P. G. Ferreira Parracho ${ }^{85}$ M. Gallinaro, ${ }^{85}$ P. Musella, ${ }^{85}$ J. Seixas, ${ }^{85}$ J. Varela,${ }^{85}$ P. Vischia, ${ }^{85}$ I. Belotelov,${ }^{86}$ P. Bunin,${ }^{86}$ I. Golutvin,${ }^{86}$ I. Gorbunov, ${ }^{86}$ V. Karjavin, ${ }^{86}$ V. Konoplyanikov, ${ }^{86}$ G. Kozlov, ${ }^{86}$ A. Lanev, ${ }^{86}$ A. Malakhov, ${ }^{86}$ P. Moisenz, ${ }^{86}$ V. Palichik, ${ }^{86}$ V. Perelygin, ${ }^{86}$ M. Savina, ${ }^{86}$ S. Shmatov, ${ }^{86}$ V. Smirnov, ${ }^{86}$ A. Volodko, ${ }^{86}$ A. Zarubin, ${ }^{86}$ S. Evstyukhin,${ }^{87}$ V. Golovtsov, ${ }^{87}$ Y. Ivanov, ${ }^{87}$ V. Kim,${ }^{87}$ P. Levchenko, ${ }^{87}$ V. Murzin, ${ }^{87}$ V. Oreshkin, ${ }^{87}$ I. Smirnov, ${ }^{87}$ V. Sulimov, ${ }^{87}$ L. Uvarov, ${ }^{87}$ S. Vavilov, ${ }^{87}$ A. Vorobyev,${ }^{87}$ An. Vorobyev, ${ }^{87}$ Yu. Andreev, ${ }^{88}$ A. Dermenev, ${ }^{88}$ S. Gninenko, ${ }^{88}$ 
N. Golubev, ${ }^{88}$ M. Kirsanov, ${ }^{88}$ N. Krasnikov, ${ }^{88}$ V. Matveev, ${ }^{88}$ A. Pashenkov, ${ }^{88}$ D. Tlisov, ${ }^{88}$ A. Toropin, ${ }^{88}$ V. Epshteyn,${ }^{89}$ M. Erofeeva, ${ }^{89}$ V. Gavrilov, ${ }^{89}$ M. Kossov, ${ }^{89, b}$ N. Lychkovskaya, ${ }^{89}$ V. Popov, ${ }^{89}$ G. Safronov, ${ }^{89}$ S. Semenov, ${ }^{89}$ V. Stolin, ${ }^{89}$ E. Vlasov, ${ }^{89}$ A. Zhokin, ${ }^{89}$ A. Belyaev,${ }^{90}$ E. Boos, ${ }^{90}$ V. Bunichev, ${ }^{90}$ M. Dubinin,${ }^{90, e}$ L. Dudko, ${ }^{90}$ A. Gribushin, ${ }^{90}$ V. Klyukhin, ${ }^{90}$ O. Kodolova, ${ }^{90}$ I. Lokhtin,,${ }^{90}$ A. Markina,${ }^{90}$ S. Obraztsov,${ }^{90}$ M. Perfilov, ${ }^{90}$ S. Petrushanko, ${ }^{90}$ L. Sarycheva, ${ }^{90, a}$ V. Savrin,,${ }^{90}$ A. Snigirev, ${ }^{90}$ V. Andreev, ${ }^{91}$ M. Azarkin, ${ }^{91}$ I. Dremin, ${ }^{91}$ M. Kirakosyan, ${ }^{91}$ A. Leonidov, ${ }^{91}$ G. Mesyats, ${ }^{91}$ S. V. Rusakov, ${ }^{91}$ A. Vinogradov,${ }^{91}$ I. Azhgirey, ${ }^{92}$ I. Bayshev, ${ }^{92}$ S. Bitioukov, ${ }^{92}$ V. Grishin, ${ }^{92, b}$ V. Kachanov,${ }^{92}$ D. Konstantinov, ${ }^{92}$ A. Korablev, ${ }^{92}$ V. Krychkine, ${ }^{92}$ V. Petrov, ${ }^{92}$ R. Ryutin, ${ }^{92}$ A. Sobol, ${ }^{92}$ L. Tourtchanovitch, ${ }^{92}$ S. Troshin,,${ }^{92}$ N. Tyurin, ${ }^{92}$ A. Uzunian, ${ }^{92}$ A. Volkov, ${ }^{92}$ P. Adzic,${ }^{93, c c}$ M. Djordjevic, ${ }^{93}$ M. Ekmedzic, ${ }^{93}$ D. Krpic, ${ }^{93, c c}$ J. Milosevic,${ }^{93}$ M. Aguilar-Benitez,${ }^{94}$ J. Alcaraz Maestre,${ }^{94}$ P. Arce, ${ }^{94}$ C. Battilana,${ }^{94}$ E. Calvo, ${ }^{94}$ M. Cerrada, ${ }^{94}$ M. Chamizo Llatas, ${ }^{94}$ N. Colino, ${ }^{94}$ B. De La Cruz,${ }^{94}$ A. Delgado Peris, ${ }^{94}$ C. Diez Pardos,${ }^{94}$ D. Domínguez Vázquez,${ }^{94}$ C. Fernandez Bedoya,${ }^{94}$ J. P. Fernández Ramos, ${ }^{94}$ A. Ferrando, ${ }^{94}$ J. Flix, ${ }^{94}$ M. C. Fouz, ${ }^{94}$ P. Garcia-Abia, ${ }^{94}$ O. Gonzalez Lopez, ${ }^{94}$ S. Goy Lopez,${ }^{94}$ J. M. Hernandez, ${ }^{94}$ M. I. Josa,${ }^{94}$ G. Merino, ${ }^{94}$ J. Puerta Pelayo, ${ }^{94}$ I. Redondo, ${ }^{94}$ L. Romero, ${ }^{94}$ J. Santaolalla, ${ }^{94}$ M. S. Soares,${ }^{94}$ C. Willmott,${ }^{94}$ C. Albajar, ${ }^{95}$ G. Codispoti, ${ }^{95}$ J. F. de Trocóniz, ${ }^{95}$ J. Cuevas, ${ }^{96}$ J. Fernandez Menendez, ${ }^{96}$ S. Folgueras, ${ }^{96}$ I. Gonzalez Caballero, ${ }^{96}$ L. Lloret Iglesias, ${ }^{96}$ J. Piedra Gomez,${ }^{96, d d}$ J. M. Vizan Garcia, ${ }^{96}$ J. A. Brochero Cifuentes, ${ }^{97}$ I. J. Cabrillo, ${ }^{97}$ A. Calderon, ${ }^{97}$ S. H. Chuang, ${ }^{97}$ J. Duarte Campderros, ${ }^{97}$ M. Felcini, ${ }^{97, \text { ee }}$ M. Fernandez, ${ }^{97}$ G. Gomez, ${ }^{97}$ J. Gonzalez Sanchez, ${ }^{97}$ C. Jorda, ${ }^{97}$ P. Lobelle Pardo, ${ }^{97}$ A. Lopez Virto, ${ }^{97}$ J. Marco, ${ }^{97}$ R. Marco, ${ }^{97}$ C. Martinez Rivero, ${ }^{97}$ F. Matorras, ${ }^{97}$ F. J. Munoz Sanchez,${ }^{97}$ T. Rodrigo,${ }^{97}$ A. Y. Rodríguez-Marrero, ${ }^{97}$ A. Ruiz-Jimeno, ${ }^{97}$ L. Scodellaro, ${ }^{97}$ M. Sobron Sanudo, ${ }^{97}$ I. Vila, ${ }^{97}$ R. Vilar Cortabitarte, ${ }^{97}$ D. Abbaneo, ${ }^{98}$ E. Auffray, ${ }^{98}$ G. Auzinger, ${ }^{98}$ P. Baillon, ${ }^{98}$ A. H. Ball, ${ }^{98}$ D. Barney,${ }^{98}$ C. Bernet, ${ }^{98, f}$ G. Bianchi, ${ }^{98}$ P. Bloch,${ }^{98}$ A. Bocci, ${ }^{98}$ A. Bonato, ${ }^{98}$ H. Breuker, ${ }^{98}$ T. Camporesi, ${ }^{98}$ G. Cerminara, ${ }^{98}$ T. Christiansen, ${ }^{98}$ J. A. Coarasa Perez,${ }^{98}$ D. D'Enterria, ${ }^{98}$ A. De Roeck, ${ }^{98}$ S. Di Guida, ${ }^{98}$ M. Dobson, ${ }^{98}$ N. Dupont-Sagorin, ${ }^{98}$ A. Elliott-Peisert, ${ }^{98}$ B. Frisch, ${ }^{98}$ W. Funk,${ }^{98}$ G. Georgiou, ${ }^{98}$ M. Giffels, ${ }^{98}$ D. Gigi,${ }^{98}$ K. Gill,${ }^{98}$ D. Giordano, ${ }^{98}$ M. Giunta, ${ }^{98}$ F. Glege,${ }^{98}$ R. Gomez-Reino Garrido, ${ }^{98}$ P. Govoni, ${ }^{98}$ S. Gowdy, ${ }^{98}$ R. Guida, ${ }^{98}$ M. Hansen, ${ }^{98}$ P. Harris,${ }^{98}$ C. Hartl, ${ }^{98}$ J. Harvey,${ }^{98}$ B. Hegner ${ }^{98}$ A. Hinzmann, ${ }^{98}$ V. Innocente, ${ }^{98}$ P. Janot,${ }^{98}$ K. Kaadze,${ }^{98}$ E. Karavakis, ${ }^{98}$ K. Kousouris, ${ }^{98}$ P. Lecoq ${ }^{98}$ P. Lenzi,${ }^{98}$ C. Lourenço, ${ }^{98}$ T. Mäki,${ }^{98}$ M. Malberti, ${ }^{98}$ L. Malgeri, ${ }^{98}$ M. Mannelli, ${ }^{98}$ L. Masetti,${ }^{98}$ F. Meijers, ${ }^{98}$ S. Mersi, ${ }^{98}$ E. Meschi, ${ }^{98}$ R. Moser,${ }^{98}$ M. U. Mozer, ${ }^{98}$ M. Mulders,${ }^{98}$ E. Nesvold,${ }^{98}$ M. Nguyen, ${ }^{98}$ T. Orimoto, ${ }^{98}$ L. Orsini, ${ }^{98}$ E. Palencia Cortezon, ${ }^{98}$ E. Perez,${ }^{98}$ A. Petrilli, ${ }^{98}$ A. Pfeiffer, ${ }^{98}$ M. Pierini, ${ }^{98}$ M. Pimiä, ${ }^{98}$ D. Piparo, ${ }^{98}$ G. Polese,${ }^{98}$ L. Quertenmont, ${ }^{98}$ A. Racz,${ }^{98}$ W. Reece, ${ }^{98}$ J. Rodrigues Antunes, ${ }^{98}$ G. Rolandi, ${ }^{98, f f}$

T. Rommerskirchen, ${ }^{98}$ C. Rovelli, ${ }^{98, g g}$ M. Rovere, ${ }^{98}$ H. Sakulin, ${ }^{98}$ F. Santanastasio, ${ }^{98}$ C. Schäfer, ${ }^{98}$ C. Schwick, ${ }^{98}$

I. Segoni, ${ }^{98}$ S. Sekmen, ${ }^{98}$ A. Sharma, ${ }^{98}$ P. Siegrist, ${ }^{98}$ P. Silva, ${ }^{98}$ M. Simon, ${ }^{98}$ P. Sphicas, ${ }^{98, \text { hh }}$ D. Spiga, ${ }^{98}$ M. Spiropulu, ${ }^{98, \mathrm{e}}$ M. Stoye, ${ }^{98}$ A. Tsirou,${ }^{98}$ G. I. Veres, ${ }^{98, \mathrm{q}}$ J. R. Vlimant, ${ }^{98}$ H. K. Wöhri, ${ }^{98}$ S. D. Worm,,${ }^{98, i i}$ W. D. Zeuner, ${ }^{98}$ W. Bertl, ${ }^{99}$ K. Deiters,${ }^{99}$ W. Erdmann, ${ }^{99}$ K. Gabathuler,${ }^{99}$ R. Horisberger, ${ }^{99}$ Q. Ingram, ${ }^{99}$ H. C. Kaestli, ${ }^{99}$ S. König, ${ }^{99}$ D. Kotlinski, ${ }^{99}$ U. Langenegger, ${ }^{99}$ F. Meier, ${ }^{99}$ D. Renker, ${ }^{99}$ T. Rohe, ${ }^{99}$ J. Sibille, ${ }^{99, j j}$ L. Bäni, ${ }^{100}$ P. Bortignon, ${ }^{100}$ M. A. Buchmann, ${ }^{100}$ B. Casal, ${ }^{100}$ N. Chanon, ${ }^{100}$ Z. Chen, ${ }^{100}$ A. Deisher, ${ }^{100}$ G. Dissertori, ${ }^{100}$ M. Dittmar, ${ }^{100}$ M. Dünser, ${ }^{100}$ J. Eugster, ${ }^{100}$ K. Freudenreich, ${ }^{100}$ C. Grab,${ }^{100}$ P. Lecomte, ${ }^{100}$ W. Lustermann, ${ }^{100}$ A. C. Marini, ${ }^{100}$ P. Martinez Ruiz del Arbol, ${ }^{100}$ N. Mohr,${ }^{100}$ F. Moortgat, ${ }^{100}$ C. Nägeli, ${ }^{100, k k}$ P. Nef, ${ }^{100}$ F. Nessi-Tedaldi, ${ }^{100}$ L. Pape,${ }^{100}$ F. Pauss,${ }^{100}$ M. Peruzzi, ${ }^{100}$ F. J. Ronga, ${ }^{100}$ M. Rossini, ${ }^{100}$ L. Sala, ${ }^{100}$

A. K. Sanchez, ${ }^{100}$ A. Starodumov, ${ }^{100,11}$ B. Stieger, ${ }^{100}$ M. Takahashi, ${ }^{100}$ L. Tauscher, ${ }^{100, a}$ A. Thea, ${ }^{100}$

K. Theofilatos, ${ }^{100}$ D. Treille, ${ }^{100}$ C. Urscheler, ${ }^{100}$ R. Wallny, ${ }^{100}$ H. A. Weber, ${ }^{100}$ L. Wehrli, ${ }^{100}$ E. Aguilo, ${ }^{101}$ C. Amsler ${ }^{101}$ V. Chiochia, ${ }^{101}$ S. De Visscher,${ }^{101}$ C. Favaro, ${ }^{101}$ M. Ivova Rikova, ${ }^{101}$ B. Millan Mejias, ${ }^{101}$ P. Otiougova, ${ }^{101}$ P. Robmann, ${ }^{101}$ H. Snoek, ${ }^{101}$ S. Tupputi, ${ }^{101}$ M. Verzetti, ${ }^{101}$ Y. H. Chang, ${ }^{102}$ K. H. Chen, ${ }^{102}$ A. Go, ${ }^{102}$ C. M. Kuo, ${ }^{102}$ S. W. Li,${ }^{102}$ W. Lin, ${ }^{102}$ Z. K. Liu, ${ }^{102}$ Y. J. Lu, ${ }^{102}$ D. Mekterovic, ${ }^{102}$ A. P. Singh,${ }^{102}$ R. Volpe ${ }^{102}$ S. S. Yu, ${ }^{102}$ P. Bartalini, ${ }^{103}$ P. Chang, ${ }^{103}$ Y. H. Chang, ${ }^{103}$ Y. W. Chang, ${ }^{103}$ Y. Chao, ${ }^{103}$ K. F. Chen, ${ }^{103}$ C. Dietz, ${ }^{103}$ U. Grundler, ${ }^{103}$ W.-S. Hou, ${ }^{103}$ Y. Hsiung, ${ }^{103}$ K. Y. Kao, ${ }^{103}$ Y. J. Lei, ${ }^{103}$ R.-S. Lu, ${ }^{103}$ D. Majumder, ${ }^{103}$ E. Petrakou, ${ }^{103}$ X. Shi, ${ }^{103}$ J. G. Shiu, ${ }^{103}$ Y. M. Tzeng, ${ }^{103}$ M. Wang, ${ }^{103}$ A. Adiguzel, ${ }^{104}$ M. N. Bakirci, ${ }^{104, m m}$ S. Cerci, ${ }^{104, n n}$ C. Dozen, ${ }^{104}$ I. Dumanoglu, ${ }^{104}$ E. Eskut, ${ }^{104}$ S. Girgis, ${ }^{104}$ G. Gokbulut, ${ }^{104}$ I. Hos, ${ }^{104}$ E. E. Kangal, ${ }^{104}$ G. Karapinar, ${ }^{104}$ A. Kayis Topaksu, ${ }^{104}$ G. Onengut, ${ }^{104}$ K. Ozdemir, ${ }^{104}$ S. Ozturk, ${ }^{104, o o}$ A. Polatoz, ${ }^{104}$ K. Sogut, ${ }^{104, p p}$

D. Sunar Cerci, ${ }^{104, \mathrm{nn}}$ B. Tali, ${ }^{104, \mathrm{nn}}$ H. Topakli, ${ }^{104, \mathrm{~mm}}$ L. N. Vergili, ${ }^{104}$ M. Vergili, ${ }^{104}$ I. V. Akin, ${ }^{105}$ T. Aliev, ${ }^{105}$ B. Bilin, ${ }^{105}$ S. Bilmis, ${ }^{105}$ M. Deniz, ${ }^{105}$ H. Gamsizkan, ${ }^{105}$ A. M. Guler, ${ }^{105}$ K. Ocalan, ${ }^{105}$ A. Ozpineci, ${ }^{105}$ M. Serin, ${ }^{105}$ R. Sever, ${ }^{105}$ U. E. Surat, ${ }^{105}$ M. Yalvac, ${ }^{105}$ E. Yildirim, ${ }^{105}$ M. Zeyrek, ${ }^{105}$ M. Deliomeroglu, ${ }^{106}$ E. Gülmez, ${ }^{106}$ 
B. Isildak, ${ }^{106}$ M. Kaya, ${ }^{106, q q}$ O. Kaya, ${ }^{106, q q}$ S. Ozkorucuklu, ${ }^{106, \text { rr }}$ N. Sonmez, ${ }^{106, s s}$ K. Cankocak, ${ }^{107}$ L. Levchuk, ${ }^{108}$ F. Bostock, ${ }^{109}$ J. J. Brooke, ${ }^{109}$ E. Clement, ${ }^{109}$ D. Cussans, ${ }^{109}$ H. Flacher, ${ }^{109}$ R. Frazier, ${ }^{109}$ J. Goldstein, ${ }^{109}$ M. Grimes, ${ }^{109}$ G. P. Heath, ${ }^{109}$ H. F. Heath,${ }^{109}$ L. Kreczko, ${ }^{109}$ S. Metson, ${ }^{109}$ D. M. Newbold, ${ }^{109, \text { ii }}$ K. Nirunpong, ${ }^{109}$ A. Poll, ${ }^{109}$ S. Senkin, ${ }^{109}$ V. J. Smith, ${ }^{109}$ T. Williams, ${ }^{109}$ L. Basso, ${ }^{110, t t}$ K. W. Bell, ${ }^{110}$ A. Belyaev, ${ }^{10, t t}$ C. Brew, ${ }^{110}$ R. M. Brown, ${ }^{110}$ D. J. A. Cockerill, ${ }^{110}$ J. A. Coughlan, ${ }^{110}$ K. Harder, ${ }^{110}$ S. Harper, ${ }^{110}$ J. Jackson, ${ }^{110}$ B. W. Kennedy, ${ }^{110}$

E. Olaiya,${ }^{110}$ D. Petyt, ${ }^{110}$ B. C. Radburn-Smith, ${ }^{110}$ C. H. Shepherd-Themistocleous, ${ }^{110}$ I. R. Tomalin, ${ }^{110}$

W. J. Womersley, ${ }^{110}$ R. Bainbridge, ${ }^{111}$ G. Ball, ${ }^{111}$ R. Beuselinck, ${ }^{111}$ O. Buchmuller, ${ }^{111}$ D. Colling, ${ }^{111}$ N. Cripps,${ }^{11}$ M. Cutajar, ${ }^{111}$ P. Dauncey, ${ }^{111}$ G. Davies,${ }^{111}$ M. Della Negra ${ }^{111}$ W. Ferguson, ${ }^{111}$ J. Fulcher, ${ }^{111}$ D. Futyan, ${ }^{111}$ A. Gilbert, ${ }^{111}$ A. Guneratne Bryer, ${ }^{11}$ G. Hall, ${ }^{111}$ Z. Hatherell, ${ }^{111}$ J. Hays, ${ }^{111}$ G. Iles, ${ }^{111}$ M. Jarvis,,${ }^{11}$

G. Karapostoli, ${ }^{111}$ L. Lyons, ${ }^{111}$ A.-M. Magnan, ${ }^{111}$ J. Marrouche, ${ }^{111}$ B. Mathias, ${ }^{111}$ R. Nandi, ${ }^{111}$ J. Nash, ${ }^{111}$ A. Nikitenko, ${ }^{111,11}$ A. Papageorgiou, ${ }^{111}$ J. Pela, ${ }^{111, \mathrm{~b}}$ M. Pesaresi, ${ }^{111}$ K. Petridis,${ }^{111}$ M. Pioppi, ${ }^{11, \text { uu }}$

D. M. Raymond, ${ }^{111}$ S. Rogerson, ${ }^{111}$ N. Rompotis, ${ }^{111}$ A. Rose, ${ }^{111}$ M. J. Ryan, ${ }^{111}$ C. Seez, ${ }^{111}$ P. Sharp, ${ }^{111, a}$ A. Sparrow, ${ }^{111}$ A. Tapper, ${ }^{111}$ M. Vazquez Acosta, ${ }^{111}$ T. Virdee, ${ }^{111}$ S. Wakefield,,${ }^{111}$ N. Wardle, ${ }^{111}$ T. Whyntie,,${ }^{111}$ M. Barrett, ${ }^{112}$ M. Chadwick, ${ }^{112}$ J. E. Cole, ${ }^{112}$ P. R. Hobson, ${ }^{112}$ A. Khan, ${ }^{112}$ P. Kyberd, ${ }^{12}$ D. Leggat,${ }^{112}$ D. Leslie, ${ }^{112}$ W. Martin, ${ }^{112}$ I. D. Reid, ${ }^{112}$ P. Symonds, ${ }^{112}$ L. Teodorescu, ${ }^{12}$ M. Turner, ${ }^{12}$ K. Hatakeyama, ${ }^{113}$ H. Liu, ${ }^{113}$ T. Scarborough, ${ }^{113}$ C. Henderson, ${ }^{114}$ P. Rumerio, ${ }^{114}$ A. Avetisyan, ${ }^{15}$ T. Bose, ${ }^{115}$ C. Fantasia, ${ }^{15}$ A. Heister, ${ }^{115}$ J. St. John, ${ }^{115}$ P. Lawson, ${ }^{115}$ D. Lazic,,${ }^{115}$ J. Rohlf, ${ }^{115}$ D. Sperka, ${ }^{115}$ L. Sulak,${ }^{115}$ J. Alimena ${ }^{116}$ S. Bhattacharya,${ }^{116}$ D. Cutts, ${ }^{116}$ A. Ferapontov, ${ }^{116}$ U. Heintz, ${ }^{116}$ S. Jabeen, ${ }^{116}$ G. Kukartsev, ${ }^{116}$ G. Landsberg, ${ }^{116}$ M. Luk, ${ }^{116}$ M. Narain, ${ }^{116}$ D. Nguyen, ${ }^{116}$ M. Segala, ${ }^{116}$ T. Sinthuprasith, ${ }^{116}$ T. Speer, ${ }^{116}$ K. V. Tsang, ${ }^{116}$ R. Breedon,,${ }^{117}$ G. Breto, ${ }^{117}$ M. Calderon De La Barca Sanchez,${ }^{117}$ S. Chauhan, ${ }^{117}$ M. Chertok, ${ }^{117}$ J. Conway, ${ }^{117}$ R. Conway, ${ }^{117}$ P. T. Cox, ${ }^{117}$ J. Dolen, ${ }^{117}$ R. Erbacher, ${ }^{117}$ M. Gardner, ${ }^{117}$ R. Houtz, ${ }^{117}$ W. Ko, ${ }^{117}$ A. Kopecky, ${ }^{117}$ R. Lander, ${ }^{117}$ O. Mall, ${ }^{117}$ T. Miceli, ${ }^{117}$ R. Nelson, ${ }^{117}$ D. Pellett,,${ }^{117}$ B. Rutherford,${ }^{117}$ M. Searle,${ }^{117}$ J. Smith, ${ }^{117}$ M. Squires, ${ }^{117}$

M. Tripathi, ${ }^{117}$ R. Vasquez Sierra, ${ }^{117}$ V. Andreev, ${ }^{118}$ D. Cline, ${ }^{118}$ R. Cousins,${ }^{118}$ J. Duris, ${ }^{118}$ S. Erhan, ${ }^{118}$ P. Everaerts, ${ }^{118}$ C. Farrell, ${ }^{118}$ J. Hauser, ${ }^{118}$ M. Ignatenko, ${ }^{118}$ C. Plager, ${ }^{118}$ G. Rakness, ${ }^{118}$ P. Schlein, ${ }^{18, a}$ J. Tucker, ${ }^{118}$ V. Valuev, ${ }^{118}$ M. Weber, ${ }^{118}$ J. Babb, ${ }^{119}$ R. Clare,${ }^{119}$ M. E. Dinardo, ${ }^{119}$ J. Ellison, ${ }^{119}$ J. W. Gary, ${ }^{119}$ F. Giordano, ${ }^{119}$

G. Hanson, ${ }^{19}$ G. Y. Jeng, ${ }^{19, v v}$ H. Liu, ${ }^{119}$ O. R. Long, ${ }^{119}$ A. Luthra, ${ }^{119}$ H. Nguyen, ${ }^{119}$ S. Paramesvaran, ${ }^{119}$ J. Sturdy, ${ }^{119}$ S. Sumowidagdo, ${ }^{119}$ R. Wilken, ${ }^{119}$ S. Wimpenny, ${ }^{119}$ W. Andrews, ${ }^{120}$ J. G. Branson, ${ }^{120}$ G. B. Cerati, ${ }^{120}$ S. Cittolin, ${ }^{120}$ D. Evans, ${ }^{120}$ F. Golf, ${ }^{120}$ A. Holzner, ${ }^{120}$ R. Kelley, ${ }^{120}$ M. Lebourgeois,${ }^{120}$ J. Letts, ${ }^{120}$ I. Macneill, ${ }^{120}$ B. Mangano, ${ }^{120}$ J. Muelmenstaedt, ${ }^{120}$ S. Padhi, ${ }^{120}$ C. Palmer, ${ }^{120}$ G. Petrucciani, ${ }^{120}$ M. Pieri, ${ }^{120}$ R. Ranieri, ${ }^{120}$ M. Sani,${ }^{120}$ V. Sharma, ${ }^{120}$ S. Simon, ${ }^{120}$ E. Sudano, ${ }^{120}$ M. Tadel,${ }^{120}$ Y. Tu, ${ }^{120}$ A. Vartak, ${ }^{120}$ S. Wasserbaech, ${ }^{120, w w}$

F. Würthwein, ${ }^{120}$ A. Yagil, ${ }^{120}$ J. Yoo, ${ }^{120}$ D. Barge, ${ }^{121}$ R. Bellan, ${ }^{121}$ C. Campagnari, ${ }^{121}$ M. D'Alfonso, ${ }^{121}$

T. Danielson, ${ }^{121}$ K. Flowers, ${ }^{121}$ P. Geffert, ${ }^{121}$ J. Incandela, ${ }^{121}$ C. Justus, ${ }^{121}$ P. Kalavase, ${ }^{121}$ S. A. Koay, ${ }^{121}$ D. Kovalskyi, ${ }^{121, \mathrm{~b}}$ V. Krutelyov, ${ }^{121}$ S. Lowette, ${ }^{121}$ N. Mccoll, ${ }^{121}$ V. Pavlunin, ${ }^{121}$ F. Rebassoo, ${ }^{121}$ J. Ribnik, ${ }^{121}$ J. Richman, ${ }^{121}$ R. Rossin, ${ }^{121}$ D. Stuart, ${ }^{121}$ W. To, ${ }^{121}$ C. West, ${ }^{121}$ A. Apresyan, ${ }^{122}$ A. Bornheim, ${ }^{122}$ Y. Chen, ${ }^{122}$ E. Di Marco, ${ }^{122}$ J. Duarte, ${ }^{122}$ M. Gataullin, ${ }^{122}$ Y. Ma, ${ }^{122}$ A. Mott, ${ }^{122}$ H. B. Newman, ${ }^{122}$ C. Rogan, ${ }^{122}$ V. Timciuc, ${ }^{122}$ P. Traczyk, ${ }^{122}$ J. Veverka, ${ }^{122}$ R. Wilkinson, ${ }^{122}$ Y. Yang, ${ }^{122}$ R. Y. Zhu, ${ }^{122}$ B. Akgun, ${ }^{123}$ R. Carroll, ${ }^{123}$ T. Ferguson, ${ }^{123}$ Y. Iiyama, ${ }^{123}$ D. W. Jang, ${ }^{123}$ Y. F. Liu, ${ }^{123}$ M. Paulini, ${ }^{123}$ H. Vogel,,${ }^{123}$ I. Vorobiev, ${ }^{123}$ J. P. Cumalat, ${ }^{124}$ B. R. Drell, ${ }^{124}$ C. J. Edelmaier, ${ }^{124}$ W. T. Ford, ${ }^{124}$ A. Gaz, ${ }^{124}$ B. Heyburn, ${ }^{124}$ E. Luiggi Lopez, ${ }^{124}$ J. G. Smith, ${ }^{124}$ K. Stenson, ${ }^{124}$ K. A. Ulmer, ${ }^{124}$ S. R. Wagner, ${ }^{124}$ L. Agostino, ${ }^{125}$ J. Alexander, ${ }^{125}$ A. Chatterjee, ${ }^{125}$ N. Eggert, ${ }^{125}$ L. K. Gibbons, ${ }^{125}$ B. Heltsley, ${ }^{125}$ W. Hopkins, ${ }^{125}$ A. Khukhunaishvili, ${ }^{125}$ B. Kreis, ${ }^{125}$ N. Mirman, ${ }^{125}$ G. Nicolas Kaufman, ${ }^{125}$

J. R. Patterson, ${ }^{125}$ A. Ryd, ${ }^{125}$ E. Salvati, ${ }^{125}$ W. Sun, ${ }^{125}$ W. D. Teo, ${ }^{125}$ J. Thom, ${ }^{125}$ J. Thompson, ${ }^{125}$ J. Vaughan, ${ }^{125}$ Y. Weng, ${ }^{125}$ L. Winstrom, ${ }^{125}$ P. Wittich, ${ }^{125}$ D. Winn, ${ }^{126}$ S. Abdullin, ${ }^{127}$ M. Albrow, ${ }^{127}$ J. Anderson, ${ }^{127}$

L. A. T. Bauerdick, ${ }^{127}$ A. Beretvas, ${ }^{127}$ J. Berryhill, ${ }^{127}$ P. C. Bhat, ${ }^{127}$ I. Bloch, ${ }^{127}$ K. Burkett, ${ }^{127}$ J. N. Butler, ${ }^{127}$ V. Chetluru, ${ }^{127}$ H. W. K. Cheung, ${ }^{127}$ F. Chlebana, ${ }^{127}$ V. D. Elvira, ${ }^{127}$ I. Fisk, ${ }^{127}$ J. Freeman, ${ }^{127}$ Y. Gao, ${ }^{127}$ D. Green, ${ }^{127}$ O. Gutsche, ${ }^{127}$ A. Hahn, ${ }^{127}$ J. Hanlon, ${ }^{127}$ R. M. Harris, ${ }^{127}$ J. Hirschauer, ${ }^{127}$ B. Hooberman, ${ }^{127}$ S. Jindariani, ${ }^{127}$ M. Johnson, ${ }^{127}$ U. Joshi, ${ }^{127}$ B. Kilminster, ${ }^{127}$ B. Klima, ${ }^{127}$ S. Kunori, ${ }^{127}$ S. Kwan, ${ }^{127}$ D. Lincoln, ${ }^{127}$ R. Lipton, ${ }^{127}$ L. Lueking, ${ }^{127}$ J. Lykken, ${ }^{127}$ K. Maeshima, ${ }^{127}$ J. M. Marraffino, ${ }^{127}$ S. Maruyama, ${ }^{127}$ D. Mason, ${ }^{127}$ P. McBride, ${ }^{127}$ K. Mishra, ${ }^{127}$ S. Mrenna, ${ }^{127}$ Y. Musienko, ${ }^{127, x x}$ C. Newman-Holmes,,${ }^{127}$ V. O’Dell, ${ }^{127}$ O. Prokofyev,${ }^{127}$

E. Sexton-Kennedy, ${ }^{127}$ S. Sharma, ${ }^{127}$ W. J. Spalding, ${ }^{127}$ L. Spiegel, ${ }^{127}$ P. Tan, ${ }^{127}$ L. Taylor, ${ }^{127}$ S. Tkaczyk,${ }^{127}$ N. V. Tran, ${ }^{127}$ L. Uplegger, ${ }^{127}$ E. W. Vaandering,${ }^{127}$ R. Vidal, ${ }^{127}$ J. Whitmore,,${ }^{127}$ W. Wu, ${ }^{127}$ F. Yang, ${ }^{127}$ F. Yumiceva, ${ }^{127}$ J. C. Yun, ${ }^{127}$ D. Acosta, ${ }^{128}$ P. Avery, ${ }^{128}$ D. Bourilkov, ${ }^{128}$ M. Chen, ${ }^{128}$ S. Das, ${ }^{128}$ M. De Gruttola, ${ }^{128}$ 
G. P. Di Giovanni, ${ }^{128}$ D. Dobur, ${ }^{128}$ A. Drozdetskiy, ${ }^{128}$ R. D. Field, ${ }^{128}$ M. Fisher, ${ }^{128}$ Y. Fu, ${ }^{128}$ I. K. Furic, ${ }^{128}$ J. Gartner, ${ }^{128}$ J. Hugon, ${ }^{128}$ B. Kim, ${ }^{128}$ J. Konigsberg, ${ }^{128}$ A. Korytov, ${ }^{128}$ A. Kropivnitskaya, ${ }^{128}$ T. Kypreos, ${ }^{128}$ J. F. Low, ${ }^{128}$ K. Matchev, ${ }^{128}$ P. Milenovic, ${ }^{128, y y}$ G. Mitselmakher, ${ }^{128}$ L. Muniz, ${ }^{128}$ R. Remington, ${ }^{128}$ A. Rinkevicius, ${ }^{128}$ P. Sellers,${ }^{128}$ N. Skhirtladze, ${ }^{128}$ M. Snowball, ${ }^{128}$ J. Yelton, ${ }^{128}$ M. Zakaria, ${ }^{128}$ V. Gaultney, ${ }^{129}$ L. M. Lebolo, ${ }^{129}$ S. Linn, ${ }^{129}$ P. Markowitz, ${ }^{129}$ G. Martinez, ${ }^{129}$ J. L. Rodriguez, ${ }^{129}$ T. Adams, ${ }^{130}$ A. Askew,${ }^{130}$ J. Bochenek, ${ }^{130}$ J. Chen, ${ }^{130}$ B. Diamond, ${ }^{130}$ S. V. Gleyzer, ${ }^{130}$ J. Haas, ${ }^{130}$ S. Hagopian, ${ }^{130}$ V. Hagopian, ${ }^{130}$ M. Jenkins, ${ }^{130}$ K. F. Johnson, ${ }^{130}$ H. Prosper, ${ }^{130}$ V. Veeraraghavan, ${ }^{130}$ M. Weinberg, ${ }^{130}$ M. M. Baarmand, ${ }^{131}$ B. Dorney, ${ }^{131}$ M. Hohlmann, ${ }^{131}$ H. Kalakhety, ${ }^{131}$ I. Vodopiyanov, ${ }^{131}$ M. R. Adams, ${ }^{132}$ I. M. Anghel,${ }^{132}$ L. Apanasevich, ${ }^{132}$ Y. Bai, ${ }^{132}$ V.E. Bazterra, ${ }^{132}$ R. R. Betts, ${ }^{132}$ J. Callner ${ }^{132}$ R. Cavanaugh, ${ }^{132}$ C. Dragoiu ${ }^{132}$ O. Evdokimov, ${ }^{132}$ E. J. Garcia-Solis, ${ }^{132}$ L. Gauthier, ${ }^{132}$ C. E. Gerber, ${ }^{132}$ D. J. Hofman, ${ }^{132}$ S. Khalatyan, ${ }^{132}$

F. Lacroix,${ }^{132}$ M. Malek, ${ }^{132}$ C. O’Brien, ${ }^{132}$ C. Silkworth, ${ }^{132}$ D. Strom,,${ }^{132}$ N. Varelas, ${ }^{132}$ U. Akgun, ${ }^{133}$ E. A. Albayrak, ${ }^{133}$ B. Bilki, ${ }^{133, z z}$ K. Chung, ${ }^{133}$ W. Clarida, ${ }^{133}$ F. Duru, ${ }^{133}$ S. Griffiths, ${ }^{133}$ C. K. Lae, ${ }^{133}$ J.-P. Merlo, ${ }^{133}$ H. Mermerkaya, ${ }^{133 \text {,aaa }}$ A. Mestvirishvili, ${ }^{133}$ A. Moeller, ${ }^{133}$ J. Nachtman, ${ }^{133}$ C. R. Newsom, ${ }^{133}$ E. Norbeck, ${ }^{133}$ J. Olson, ${ }^{133}$ Y. Onel, ${ }^{133}$ F. Ozok, ${ }^{133}$ S. Sen, ${ }^{133}$ E. Tiras, ${ }^{133}$ J. Wetzel, ${ }^{133}$ T. Yetkin, ${ }^{133}$ K. Yi, ${ }^{133}$ B. A. Barnett, ${ }^{134}$ B. Blumenfeld, ${ }^{134}$ S. Bolognesi, ${ }^{134}$ D. Fehling, ${ }^{134}$ G. Giurgiu, ${ }^{134}$ A. V. Gritsan, ${ }^{134}$ Z. J. Guo, ${ }^{134}$ G. Hu, ${ }^{134}$ P. Maksimovic, ${ }^{134}$ S. Rappoccio, ${ }^{134}$ M. Swartz, ${ }^{134}$ A. Whitbeck,${ }^{134}$ P. Baringer, ${ }^{135}$ A. Bean, ${ }^{135}$ G. Benelli, ${ }^{135}$ O. Grachov, ${ }^{135}$ R. P. Kenny Iii,,${ }^{135}$ M. Murray, ${ }^{135}$ D. Noonan, ${ }^{135}$ V. Radicci, ${ }^{135}$ S. Sanders, ${ }^{135}$ R. Stringer, ${ }^{135}$ G. Tinti, ${ }^{135}$ J. S. Wood, ${ }^{135}$ V. Zhukova, ${ }^{135}$ A. F. Barfuss, ${ }^{136}$ T. Bolton, ${ }^{136}$ I. Chakaberia, ${ }^{136}$ A. Ivanov, ${ }^{136}$ S. Khalil, ${ }^{136}$ M. Makouski, ${ }^{136}$ Y. Maravin, ${ }^{136}$ S. Shrestha, ${ }^{136}$ I. Svintradze, ${ }^{136}$ J. Gronberg, ${ }^{137}$ D. Lange, ${ }^{137}$ D. Wright, ${ }^{137}$ A. Baden, ${ }^{138}$ M. Boutemeur, ${ }^{138}$ B. Calvert, ${ }^{138}$ S. C. Eno, ${ }^{138}$ J. A. Gomez,${ }^{138}$ N. J. Hadley, ${ }^{138}$ R. G. Kellogg, ${ }^{138}$ M. Kirn, ${ }^{138}$ T. Kolberg, ${ }^{138}$ Y. Lu, ${ }^{138}$ M. Marionneau, ${ }^{138}$ A. C. Mignerey, ${ }^{138}$ A. Peterman, ${ }^{138}$ K. Rossato, ${ }^{138}$ A. Skuja, ${ }^{138}$ J. Temple, ${ }^{138}$ M. B. Tonjes, ${ }^{138}$ S. C. Tonwar, ${ }^{138}$ E. Twedt, ${ }^{138}$ G. Bauer, ${ }^{139}$ J. Bendavid, ${ }^{139}$ W. Busza, ${ }^{139}$ E. Butz, ${ }^{139}$ I. A. Cali, ${ }^{139}$ M. Chan, ${ }^{139}$ V. Dutta, ${ }^{139}$ G. Gomez Ceballos, ${ }^{139}$ M. Goncharov, ${ }^{139}$ K. A. Hahn, ${ }^{139}$ Y. Kim, ${ }^{139}$ M. Klute, ${ }^{139}$ Y.-J. Lee, ${ }^{139}$ W. Li, ${ }^{139}$ P. D. Luckey, ${ }^{139}$ T. Ma, ${ }^{139}$ S. Nahn, ${ }^{139}$ C. Paus,${ }^{139}$ D. Ralph,${ }^{139}$ C. Roland ${ }^{139}$ G. Roland, ${ }^{139}$ M. Rudolph, ${ }^{139}$ G. S. F. Stephans, ${ }^{139}$ F. Stöckli, ${ }^{139}$ K. Sumorok, ${ }^{139}$ K. Sung, ${ }^{139}$ D. Velicanu, ${ }^{139}$ E. A. Wenger, ${ }^{139}$ R. Wolf, ${ }^{139}$ B. Wyslouch, ${ }^{139}$ S. Xie, ${ }^{139}$ M. Yang, ${ }^{139}$ Y. Yilmaz, ${ }^{139}$ A. S. Yoon, ${ }^{139}$ M. Zanetti, ${ }^{139}$ S. I. Cooper, ${ }^{140}$ P. Cushman, ${ }^{140}$ B. Dahmes, ${ }^{140}$ A. De Benedetti,${ }^{140}$ G. Franzoni, ${ }^{140}$ A. Gude, ${ }^{140}$ J. Haupt,${ }^{140}$ S. C. Kao, ${ }^{140}$ K. Klapoetke, ${ }^{140}$ Y. Kubota, ${ }^{140}$ J. Mans, ${ }^{140}$ N. Pastika, ${ }^{140}$ R. Rusack,${ }^{140}$ M. Sasseville,${ }^{140}$ A. Singovsky ${ }_{140}$ N. Tambe, ${ }^{140}$ J. Turkewitz ${ }^{140}$ L. M. Cremaldi, ${ }^{141}$ R. Kroeger, ${ }^{141}$ L. Perera, ${ }^{141}$ R. Rahmat, ${ }^{141}$ D. A. Sanders ${ }^{141}$ E. Avdeeva, ${ }^{142}$ K. Bloom, ${ }^{142}$ S. Bose,${ }^{142}$ J. Butt, ${ }^{142}$ D. R. Claes, ${ }^{142}$ A. Dominguez, ${ }^{142}$ M. Eads,${ }^{142}$ P. Jindal, ${ }^{142}$ J. Keller, ${ }^{142}$ I. Kravchenko, ${ }^{142}$ J. Lazo-Flores, ${ }^{142}$ H. Malbouisson, ${ }^{142}$ S. Malik, ${ }^{142}$ G. R. Snow, ${ }^{142}$ U. Baur, ${ }^{143}$ A. Godshalk, ${ }^{143}$ I. Iashvili, ${ }^{143}$ S. Jain, ${ }^{143}$ A. Kharchilava, ${ }^{143}$ A. Kumar, ${ }^{143}$ S. P. Shipkowski, ${ }^{143}$ K. Smith, ${ }^{143}$ G. Alverson, ${ }^{144}$ E. Barberis,${ }^{144}$ D. Baumgartel, ${ }^{144}$ M. Chasco,${ }^{144}$ J. Haley, ${ }^{144}$ D. Trocino, ${ }^{144}$ D. Wood, ${ }^{144}$ J. Zhang, ${ }^{144}$ A. Anastassov, ${ }^{145}$ A. Kubik, ${ }^{145}$ N. Mucia, ${ }^{145}$ N. Odell, ${ }^{145}$ R. A. Ofierzynski, ${ }^{145}$ B. Pollack, ${ }^{145}$ A. Pozdnyakov, ${ }^{145}$ M. Schmitt, ${ }^{145}$ S. Stoynev, ${ }^{145}$ M. Velasco, ${ }^{145}$ S. Won, ${ }^{145}$ L. Antonelli,,${ }^{146}$ D. Berry, ${ }^{146}$ A. Brinkerhoff, ${ }^{146}$ M. Hildreth, ${ }^{146}$ C. Jessop, ${ }^{146}$ D. J. Karmgard, ${ }^{146}$ J. Kolb, ${ }^{146}$ K. Lannon, ${ }^{146}$ W. Luo, ${ }^{146}$ S. Lynch, ${ }^{146}$ N. Marinelli, ${ }^{146}$ D. M. Morse, ${ }^{146}$ T. Pearson, ${ }^{146}$ R. Ruchti, ${ }^{146}$ J. Slaunwhite, ${ }^{146}$ N. Valls, ${ }^{146}$ J. Warchol, ${ }^{146}$ M. Wayne, ${ }^{146}$ M. Wolf, ${ }^{146}$ J. Ziegler, ${ }^{146}$ B. Bylsma, ${ }^{147}$ L. S. Durkin, ${ }^{147}$ C. Hill, ${ }^{147}$ R. Hughes, ${ }^{147}$ P. Killewald ${ }^{147}$ K. Kotov, ${ }^{147}$ T. Y. Ling, ${ }^{147}$ D. Puigh, ${ }^{147}$ M. Rodenburg,,${ }^{147}$ C. Vuosalo, ${ }^{147}$ G. Williams, ${ }^{147}$ B. L. Winer, ${ }^{147}$ N. Adam, ${ }^{148}$ E. Berry,${ }^{148}$ P. Elmer, ${ }^{148}$ D. Gerbaudo ${ }^{148}$ V. Halyo, ${ }^{148}$ P. Hebda, ${ }^{148}$ J. Hegeman, ${ }^{148}$ A. Hunt, ${ }^{148}$ E. Laird, ${ }^{148}$ D. Lopes Pegna, ${ }^{148}$ P. Lujan, ${ }^{148}$ D. Marlow, ${ }^{148}$ T. Medvedeva, ${ }^{148}$ M. Mooney,${ }^{148}$ J. Olsen, ${ }^{148}$ P. Piroué, ${ }^{148}$ X. Quan, ${ }^{148}$ A. Raval,${ }^{148}$ H. Saka, ${ }^{148}$ D. Stickland, ${ }^{148}$ C. Tully, ${ }^{148}$ J. S. Werner, ${ }^{148}$ A. Zuranski, ${ }^{148}$ J. G. Acosta, ${ }^{149}$ X. T. Huang, ${ }^{149}$ A. Lopez,${ }^{149}$ H. Mendez, ${ }^{149}$ S. Oliveros, ${ }^{149}$ J. E. Ramirez Vargas, ${ }^{149}$ A. Zatserklyaniy, ${ }^{149}$ E. Alagoz, ${ }^{150}$ V.E. Barnes, ${ }^{150}$ D. Benedetti, ${ }^{150}$ G. Bolla, ${ }^{150}$ D. Bortoletto, ${ }^{150}$ M. De Mattia, ${ }^{150}$ A. Everett, ${ }^{150}$ Z. Hu, ${ }^{150}$ M. Jones, ${ }^{150}$ O. Koybasi, ${ }^{150}$ M. Kress, ${ }^{150}$ A. T. Laasanen, ${ }^{150}$ N. Leonardo, ${ }^{150}$ V. Maroussov, ${ }^{150}$ P. Merkel, ${ }^{150}$ D. H. Miller, ${ }^{150}$ N. Neumeister, ${ }^{150}$ I. Shipsey, ${ }^{150}$ D. Silvers, ${ }^{150}$ A. Svyatkovskiy, ${ }^{150}$ M. Vidal Marono, ${ }^{150}$ H. D. Yoo, ${ }^{150}$ J. Zablocki, ${ }^{150}$ Y. Zheng, ${ }^{150}$ S. Guragain, ${ }^{151}$ N. Parashar, ${ }^{151}$ A. Adair, ${ }^{152}$ C. Boulahouache, ${ }^{152}$ V. Cuplov, ${ }^{152}$ K. M. Ecklund, ${ }^{152}$ F. J. M. Geurts, ${ }^{152}$ B. P. Padley, ${ }^{152}$ R. Redjimi, ${ }^{152}$ J. Roberts, ${ }^{152}$ J. Zabel, ${ }^{152}$ B. Betchart, ${ }^{153}$ A. Bodek, ${ }^{153}$ Y. S. Chung,,${ }^{153}$ R. Covarelli, ${ }^{153}$ P. de Barbaro, ${ }^{153}$ R. Demina, ${ }^{153}$ Y. Eshaq,${ }^{153}$ A. Garcia-Bellido, ${ }^{153}$ P. Goldenzweig, ${ }^{153}$ Y. Gotra, ${ }^{153}$ J. Han, ${ }^{153}$ A. Harel, ${ }^{153}$ S. Korjenevski, ${ }^{153}$ D. C. Miner, ${ }^{153}$ D. Vishnevskiy, ${ }^{153}$ M. Zielinski, ${ }^{153}$ A. Bhatti,${ }^{154}$ R. Ciesielski, ${ }^{154}$ L. Demortier, ${ }^{154}$ K. Goulianos, ${ }^{154}$ G. Lungu, ${ }^{154}$ 
S. Malik, ${ }^{154}$ C. Mesropian, ${ }^{154}$ S. Arora, ${ }^{155}$ A. Barker, ${ }^{155}$ J. P. Chou, ${ }^{155}$ C. Contreras-Campana, ${ }^{155}$ E. Contreras-Campana, ${ }^{155}$ D. Duggan, ${ }^{155}$ D. Ferencek,${ }^{155}$ Y. Gershtein, ${ }^{155}$ R. Gray,${ }^{155}$ E. Halkiadakis, ${ }^{155}$ D. Hidas, ${ }^{155}$ D. Hits, ${ }^{155}$ A. Lath, ${ }^{155}$ S. Panwalkar, ${ }^{155}$ M. Park, ${ }^{155}$ R. Patel, ${ }^{155}$ V. Rekovic, ${ }^{155}$ A. Richards, ${ }^{155}$ J. Robles, ${ }^{155}$ K. Rose, ${ }^{155}$ S. Salur, ${ }^{155}$ S. Schnetzer, ${ }^{155}$ C. Seitz, ${ }^{155}$ S. Somalwar, ${ }^{155}$ R. Stone, ${ }^{155}$ S. Thomas, ${ }^{155}$ G. Cerizza, ${ }^{156}$ M. Hollingsworth, ${ }^{156}$ S. Spanier, ${ }^{156}$ Z. C. Yang, ${ }^{156}$ A. York, ${ }^{156}$ R. Eusebi,,${ }^{157}$ W. Flanagan, ${ }^{157}$ J. Gilmore, ${ }^{157}$ T. Kamon, ${ }^{157, b b b}$ V. Khotilovich, ${ }^{157}$ R. Montalvo, ${ }^{157}$ I. Osipenkov, ${ }^{157}$ Y. Pakhotin, ${ }^{157}$ A. Perloff,,${ }^{157}$ J. Roe, ${ }^{157}$ A. Safonov, ${ }^{157}$ T. Sakuma, ${ }^{157}$ S. Sengupta, ${ }^{157}$ I. Suarez, ${ }^{157}$ A. Tatarinov, ${ }^{157}$ D. Toback,${ }^{157}$ N. Akchurin, ${ }^{158}$ J. Damgov, ${ }^{158}$ P. R. Dudero, ${ }^{158}$ C. Jeong, ${ }^{158}$ K. Kovitanggoon, ${ }^{158}$ S. W. Lee, ${ }^{158}$ T. Libeiro, ${ }^{158}$ Y. Roh, ${ }^{158}$ I. Volobouev, ${ }^{158}$ E. Appelt, ${ }^{159}$ D. Engh, ${ }^{159}$ C. Florez, ${ }^{159}$ S. Greene, ${ }^{159}$ A. Gurrola, ${ }^{159}$ W. Johns, ${ }^{159}$ P. Kurt, ${ }^{159}$ C. Maguire, ${ }^{159}$ A. Melo, ${ }^{159}$ P. Sheldon, ${ }^{159}$ B. Snook, ${ }^{159}$ S. Tuo, ${ }^{159}$ J. Velkovska, ${ }^{159}$ M. W. Arenton, ${ }^{160}$ M. Balazs, ${ }^{160}$ S. Boutle,${ }^{160}$ B. Cox ${ }^{160}$ B. Francis,${ }^{160}$ J. Goodell, ${ }^{160}$ R. Hirosky, ${ }^{160}$ A. Ledovskoy, ${ }^{160}$ C. Lin, ${ }^{160}$ C. Neu, ${ }^{160}$ J. Wood, ${ }^{160}$ R. Yohay, ${ }^{160}$ S. Gollapinni, ${ }^{161}$ R. Harr, ${ }^{161}$ P. E. Karchin, ${ }^{161}$ C. Kottachchi Kankanamge Don, ${ }^{161}$ P. Lamichhane, ${ }^{161}$ A. Sakharov, ${ }^{161}$ M. Anderson, ${ }^{162}$ M. Bachtis, ${ }^{162}$ D. Belknap, ${ }^{162}$ L. Borrello, ${ }^{162}$ D. Carlsmith, ${ }^{162}$ M. Cepeda, ${ }^{162}$ S. Dasu, ${ }^{162}$ L. Gray, ${ }^{162}$ K. S. Grogg, ${ }^{162}$ M. Grothe, ${ }^{162}$ R. Hall-Wilton, ${ }^{162}$ M. Herndon, ${ }^{162}$ A. Hervé, ${ }^{162}$ P. Klabbers, ${ }^{162}$ J. Klukas, ${ }^{162}$ A. Lanaro, ${ }^{162}$ C. Lazaridis, ${ }^{162}$ J. Leonard, ${ }^{162}$ R. Loveless, ${ }^{162}$ A. Mohapatra, ${ }^{162}$ I. Ojalvo, ${ }^{162}$ G. A. Pierro, ${ }^{162}$ I. Ross, ${ }^{162}$ A. Savin, ${ }^{162}$ W. H. Smith, ${ }^{162}$ and J. Swanson ${ }^{162}$

\title{
(CMS Collaboration)
}

\author{
${ }^{1}$ Yerevan Physics Institute, Yerevan, Armenia \\ ${ }^{2}$ Institut für Hochenergiephysik der OeAW, Wien, Austria \\ ${ }^{3}$ National Centre for Particle and High Energy Physics, Minsk, Belarus \\ ${ }^{4}$ Universiteit Antwerpen, Antwerpen, Belgium \\ ${ }^{5}$ Vrije Universiteit Brussel, Brussel, Belgium \\ ${ }^{6}$ Université Libre de Bruxelles, Bruxelles, Belgium \\ ${ }^{7}$ Ghent University, Ghent, Belgium \\ ${ }^{8}$ Université Catholique de Louvain, Louvain-la-Neuve, Belgium \\ ${ }^{9}$ Université de Mons, Mons, Belgium \\ ${ }^{10}$ Centro Brasileiro de Pesquisas Fisicas, Rio de Janeiro, Brazil \\ ${ }^{11}$ Universidade do Estado do Rio de Janeiro, Rio de Janeiro, Brazil \\ ${ }^{12}$ Instituto de Fisica Teorica, Universidade Estadual Paulista, Sao Paulo, Brazil \\ ${ }^{13}$ Institute for Nuclear Research and Nuclear Energy, Sofia, Bulgaria \\ ${ }^{14}$ University of Sofia, Sofia, Bulgaria \\ ${ }^{15}$ Institute of High Energy Physics, Beijing, China \\ ${ }^{16}$ State Key Lab. of Nucl. Phys. and Tech., Peking University, Beijing, China \\ ${ }^{17}$ Universidad de Los Andes, Bogota, Colombia \\ ${ }^{18}$ Technical University of Split, Split, Croatia \\ ${ }^{19}$ University of Split, Split, Croatia \\ ${ }^{20}$ Institute Rudjer Boskovic, Zagreb, Croatia \\ ${ }^{21}$ University of Cyprus, Nicosia, Cyprus \\ ${ }^{22}$ Charles University, Prague, Czech Republic \\ ${ }^{23}$ Academy of Scientific Research and Technology of the Arab Republic of Egypt, \\ Egyptian Network of High Energy Physics, Cairo, Egypt \\ ${ }^{24}$ National Institute of Chemical Physics and Biophysics, Tallinn, Estonia \\ ${ }^{25}$ Department of Physics, University of Helsinki, Helsinki, Finland \\ ${ }^{26}$ Helsinki Institute of Physics, Helsinki, Finland \\ ${ }^{27}$ Lappeenranta University of Technology, Lappeenranta, Finland \\ ${ }^{28}$ DSM/IRFU, CEA/Saclay, Gif-sur-Yvette, France \\ ${ }^{29}$ Laboratoire Leprince-Ringuet, Ecole Polytechnique, IN2P3-CNRS, Palaiseau, France \\ ${ }^{30}$ Institut Pluridisciplinaire Hubert Curien, Université de Strasbourg, \\ Université de Haute-Alsace Mulhouse, CNRS/IN2P3, Strasbourg, France \\ ${ }^{31}$ Centre de Calcul de l'Institut National de Physique Nucleaire et de Physique des Particules (IN2P3), Villeurbanne, France \\ ${ }^{32}$ Université de Lyon, Université Claude Bernard Lyon 1, CNRS-IN2P3, Institut de Physique Nucléaire de Lyon, Villeurbanne, France \\ ${ }^{33}$ Institute of High Energy Physics and Informatization, Tbilisi State University, Tbilisi, Georgia \\ ${ }^{34}$ RWTH Aachen University, I. Physikalisches Institut, Aachen, Germany \\ ${ }^{35}$ RWTH Aachen University, III. Physikalisches Institut A, Aachen, Germany \\ ${ }^{36}$ RWTH Aachen University, III. Physikalisches Institut B, Aachen, Germany
}


${ }^{37}$ Deutsches Elektronen-Synchrotron, Hamburg, Germany

${ }^{38}$ University of Hamburg, Hamburg, Germany

${ }^{39}$ Institut für Experimentelle Kernphysik, Karlsruhe, Germany

${ }^{40}$ Institute of Nuclear Physics “Demokritos,” Aghia Paraskevi, Greece

${ }^{41}$ University of Athens, Athens, Greece

${ }^{42}$ University of Ioánnina, Ioánnina, Greece

${ }^{43}$ KFKI Research Institute for Particle and Nuclear Physics, Budapest, Hungary

${ }^{44}$ Institute of Nuclear Research ATOMKI, Debrecen, Hungary

${ }^{45}$ University of Debrecen, Debrecen, Hungary

${ }^{46}$ Panjab University, Chandigarh, India

${ }^{47}$ University of Delhi, Delhi, India

${ }^{48}$ Saha Institute of Nuclear Physics, Kolkata, India

${ }^{49}$ Bhabha Atomic Research Centre, Mumbai, India

${ }^{50}$ Tata Institute of Fundamental Research-EHEP, Mumbai, India

${ }^{51}$ Tata Institute of Fundamental Research-HECR, Mumbai, India

${ }^{52}$ Institute for Research in Fundamental Sciences (IPM), Tehran, Iran

${ }^{53 a}$ INFN Sezione di Bari, Bari, Italy

${ }^{53 \mathrm{~b}}$ Università di Bari, Bari, Italy

${ }^{53 \mathrm{c}}$ Politecnico di Bari, Bari, Italy

${ }^{54 a}$ INFN Sezione di Bologna, Bologna, Italy

${ }^{54 \mathrm{~b}}$ Università di Bologna, Bologna, Italy

${ }^{55 a}$ INFN Sezione di Catania, Catania, Italy

${ }^{55 \mathrm{~b}}$ Università di Catania, Catania, Italy

${ }^{56 a}$ INFN Sezione di Firenze, Firenze, Italy

${ }^{56 \mathrm{~b}}$ Università di Firenze, Firenze, Italy

${ }^{57}$ INFN Laboratori Nazionali di Frascati, Frascati, Italy

${ }^{58}$ INFN Sezione di Genova, Genova, Italy

${ }^{59 a}$ INFN Sezione di Milano-Bicocca, Milano, Italy

${ }^{59 b}$ Università di Milano-Bicocca, Milano, Italy

${ }^{60 a}$ INFN Sezione di Napoli, Napoli, Italy

${ }^{60 \mathrm{~b}}$ Università di Napoli "Federico II", Napoli, Italy

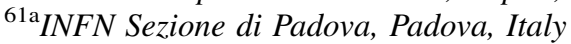

${ }^{61 \mathrm{~b}}$ Università di Padova, Padova, Italy

${ }^{61 \mathrm{c}}$ Università di Trento (Trento), Padova, Italy

${ }^{62 \mathrm{a}}$ INFN Sezione di Pavia, Pavia, Italy

${ }^{62 \mathrm{~b}}$ Università di Pavia, Pavia, Italy

${ }^{63 a}$ INFN Sezione di Perugia, Perugia, Italy

${ }^{63 \mathrm{~b}}$ Università di Perugia, Perugia, Italy

${ }^{64 \mathrm{a}}$ INFN Sezione di Pisa, Pisa, Italy

${ }^{64 \mathrm{~b}}$ Università di Pisa, Pisa, Italy

${ }^{64 \mathrm{c}}$ Scuola Normale Superiore di Pisa, Pisa, Italy

${ }^{65}$ INFN Sezione di Roma, Roma, Italy

${ }^{65 b}$ Università di Roma "La Sapienza”, Roma, Italy

${ }^{66 \mathrm{a}}$ INFN Sezione di Torino, Torino, Italy

${ }^{66 \mathrm{~b}}$ Università di Torino, Torino, Italy

${ }^{66 \mathrm{c}}$ Università del Piemonte Orientale (Novara), Torino, Italy

${ }^{67}$ INFN Sezione di Trieste, Trieste, Italy

${ }^{67 \mathrm{~b}}$ Università di Trieste, Trieste, Italy

${ }^{68}$ Kangwon National University, Chunchon, Korea

${ }^{69}$ Kyungpook National University, Daegu, Korea

${ }^{70}$ Chonnam National University, Institute for Universe and Elementary Particles, Kwangju, Korea

${ }^{71}$ Konkuk University, Seoul, Korea

${ }^{72}$ Korea University, Seoul, Korea

${ }^{73}$ University of Seoul, Seoul, Korea

${ }^{74}$ Sungkyunkwan University, Suwon, Korea

${ }^{75}$ Vilnius University, Vilnius, Lithuania

${ }^{76}$ Centro de Investigacion y de Estudios Avanzados del IPN, Mexico City, Mexico

${ }^{77}$ Universidad Iberoamericana, Mexico City, Mexico

${ }^{78}$ Benemerita Universidad Autonoma de Puebla, Puebla, Mexico

${ }^{79}$ Universidad Autónoma de San Luis Potosí, San Luis Potosí, Mexico

${ }^{80}$ University of Auckland, Auckland, New Zealand 
${ }^{81}$ University of Canterbury, Christchurch, New Zealand

${ }^{82}$ National Centre for Physics, Quaid-I-Azam University, Islamabad, Pakistan

${ }^{83}$ Institute of Experimental Physics, Faculty of Physics, University of Warsaw, Warsaw, Poland

${ }^{84}$ Soltan Institute for Nuclear Studies, Warsaw, Poland

${ }^{85}$ Laboratório de Instrumentação e Física Experimental de Partículas, Lisboa, Portugal

${ }^{86}$ Joint Institute for Nuclear Research, Dubna, Russia

${ }^{87}$ Petersburg Nuclear Physics Institute, Gatchina (St Petersburg), Russia

${ }^{88}$ Institute for Nuclear Research, Moscow, Russia

${ }^{89}$ Institute for Theoretical and Experimental Physics, Moscow, Russia

${ }^{90}$ Moscow State University, Moscow, Russia

${ }^{91}$ P.N. Lebedev Physical Institute, Moscow, Russia

${ }^{92}$ State Research Center of Russian Federation, Institute for High Energy Physics, Protvino, Russia

${ }^{93}$ University of Belgrade, Faculty of Physics and Vinca Institute of Nuclear Sciences, Belgrade, Serbia

${ }^{94}$ Centro de Investigaciones Energéticas Medioambientales y Tecnológicas (CIEMAT), Madrid, Spain

${ }^{95}$ Universidad Autónoma de Madrid, Madrid, Spain

${ }^{96}$ Universidad de Oviedo, Oviedo, Spain

${ }^{97}$ Instituto de Física de Cantabria (IFCA), CSIC-Universidad de Cantabria, Santander, Spain

${ }^{98}$ CERN, European Organization for Nuclear Research, Geneva, Switzerland

${ }^{99}$ Paul Scherrer Institut, Villigen, Switzerland

${ }^{100}$ Institute for Particle Physics, ETH Zurich, Zurich, Switzerland

${ }^{101}$ Universität Zürich, Zurich, Switzerland

${ }^{102}$ National Central University, Chung-Li, Taiwan

${ }^{103}$ National Taiwan University (NTU), Taipei, Taiwan

${ }^{104}$ Cukurova University, Adana, Turkey

${ }^{105}$ Middle East Technical University, Physics Department, Ankara, Turkey

${ }^{106}$ Bogazici University, Istanbul, Turkey

${ }^{107}$ Istanbul Technical University, Istanbul, Turkey

${ }^{108}$ National Scientific Center, Kharkov Institute of Physics and Technology, Kharkov, Ukraine

${ }^{109}$ University of Bristol, Bristol, United Kingdom

${ }^{110}$ Rutherford Appleton Laboratory, Didcot, United Kingdom

${ }^{111}$ Imperial College, London, United Kingdom

${ }^{112}$ Brunel University, Uxbridge, United Kingdom

${ }^{113}$ Baylor University, Waco, Texas, USA

${ }^{114}$ The University of Alabama, Tuscaloosa, Alabama, USA

${ }^{115}$ Boston University, Boston, Massachusetts, USA

${ }^{116}$ Brown University, Providence, Rhode Island, USA

${ }^{117}$ University of California, Davis, Davis, California, USA

${ }^{118}$ University of California, Los Angeles, Los Angeles, California, USA

${ }^{119}$ University of California, Riverside, Riverside, California, USA

${ }^{120}$ University of California, San Diego, La Jolla, California, USA

${ }^{121}$ University of California, Santa Barbara, Santa Barbara, California, USA

${ }^{122}$ California Institute of Technology, Pasadena, California, USA

${ }^{123}$ Carnegie Mellon University, Pittsburgh, Pennsylvania, USA

${ }^{124}$ University of Colorado at Boulder, Boulder, Colorado, USA

${ }^{125}$ Cornell University, Ithaca, New York, USA

${ }^{126}$ Fairfield University, Fairfield, Connecticut, USA

${ }^{127}$ Fermi National Accelerator Laboratory, Batavia, Illionois, USA

${ }^{128}$ University of Florida, Gainesville, Florida, USA

${ }^{129}$ Florida International University, Miami, Florida, USA

${ }^{130}$ Florida State University, Tallahassee, Florida, USA

${ }^{131}$ Florida Institute of Technology, Melbourne, Florida, USA

${ }^{132}$ University of Illinois at Chicago (UIC), Chicago, Illinois, USA

${ }^{133}$ The University of Iowa, Iowa City, Iowa, USA

${ }^{134}$ Johns Hopkins University, Baltimore, Maryland, USA

${ }^{135}$ The University of Kansas, Lawrence, Kansas, USA

${ }^{136}$ Kansas State University, Manhattan, Kansas, USA

${ }^{137}$ Lawrence Livermore National Laboratory, Livermore, California, USA

${ }^{138}$ University of Maryland, College Park, Maryland, USA

${ }^{139}$ Massachusetts Institute of Technology, Cambridge, Massachusetts, USA

${ }^{140}$ University of Minnesota, Minneapolis, Minnesota, USA

${ }^{141}$ University of Mississippi, University, Mississippi, USA 


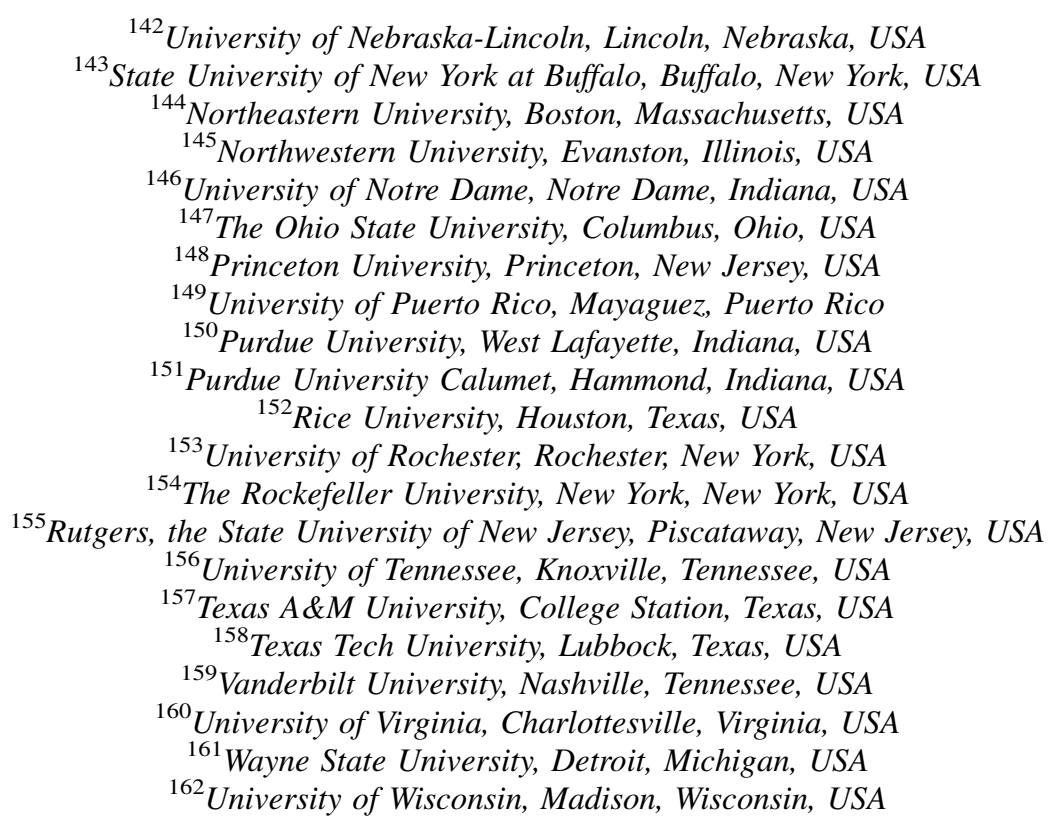

${ }^{\mathrm{a}}$ Deceased.

${ }^{\mathrm{b}}$ Also at CERN, European Organization for Nuclear Research, Geneva, Switzerland.

${ }^{\mathrm{c}}$ Also at National Institute of Chemical Physics and Biophysics, Tallinn, Estonia.

${ }^{\mathrm{d}}$ Also at Universidade Federal do ABC, Santo Andre, Brazil.

${ }^{\mathrm{e}}$ Also at California Institute of Technology, Pasadena, California, USA.

${ }^{\mathrm{f}}$ Also at Laboratoire Leprince-Ringuet, Ecole Polytechnique, IN2P3-CNRS, Palaiseau, France.

${ }^{\mathrm{g}}$ Also at Suez Canal University, Suez, Egypt.

${ }^{\mathrm{h}}$ Also at Zewail City of Science and Technology, Zewail, Egypt.

${ }^{\mathrm{i}}$ Also at Cairo University, Cairo, Egypt.

${ }^{\mathrm{j}}$ Also at British University, Cairo, Egypt.

${ }^{\mathrm{k}}$ Also at Fayoum University, El-Fayoum, Egypt.

${ }^{1}$ Also at Soltan Institute for Nuclear Studies, Warsaw, Poland.

${ }^{\mathrm{m}}$ Also at Université de Haute-Alsace, Mulhouse, France.

${ }^{\mathrm{n}}$ Also at Moscow State University, Moscow, Russia.

${ }^{\circ}$ Also at Brandenburg University of Technology, Cottbus, Germany.

${ }^{\mathrm{p}}$ Also at Institute of Nuclear Research ATOMKI, Debrecen, Hungary.

${ }^{\mathrm{q}}$ Also at Eötvös Loránd University, Budapest, Hungary.

${ }^{\mathrm{r}}$ Also at Tata Institute of Fundamental Research-HECR, Mumbai, India.

${ }^{\mathrm{s}}$ Also at University of Visva-Bharati, Santiniketan, India.

${ }^{\mathrm{t}}$ Also at Sharif University of Technology, Tehran, Iran.

${ }^{\mathrm{u}}$ Also at Isfahan University of Technology, Isfahan, Iran.

${ }^{\mathrm{v}}$ Also at Shiraz University, Shiraz, Iran.

${ }^{\mathrm{w}}$ Also at Plasma Physics Research Center, Science and Research Branch, Islamic Azad University, Teheran, Iran.

${ }^{x}$ Also at Facoltà Ingegneria Università di Roma, Roma, Italy.

${ }^{y}$ Also at Università della Basilicata, Potenza, Italy.

${ }^{\mathrm{z}}$ Also at Università degli Studi Guglielmo Marconi, Roma, Italy.

${ }^{\text {aa } A l s o ~ a t ~ U n i v e r s i t a ̀ ~ d e g l i ~ s t u d i ~ d i ~ S i e n a, ~ S i e n a, ~ I t a l y . ~}$

${ }^{\mathrm{bb}}$ Also at University of Bucharest, Bucuresti-Magurele, Romania.

${ }^{\mathrm{cc}}$ Also at Faculty of Physics of University of Belgrade, Belgrade, Serbia.

${ }^{\mathrm{dd}}$ Also at University of Florida, Gainesville, USA.

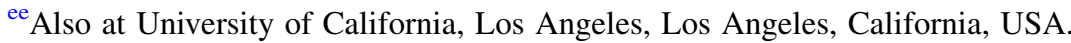

${ }^{\mathrm{ff}}$ Also at Scuola Normale e Sezione dell' INFN, Pisa, Italy,

gg Also at INFN Sezione di Roma, Università di Roma "La Sapienza", Roma, Italy.

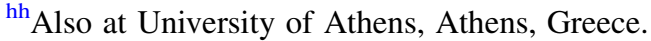

${ }^{i i}$ Also at Rutherford Appleton Laboratory, Didcot, United Kingdom. 
${ }^{\mathrm{jj}}$ Also at The University of Kansas, Lawrence, Kansas, USA.

${ }^{\mathrm{kk}}$ Also at Paul Scherrer Institut, Villigen, Switzerland.

${ }^{11}$ Also at Institute for Theoretical and Experimental Physics, Moscow, Russia.

${ }^{\mathrm{mm}}$ Also at Gaziosmanpasa University, Tokat, Turkey.

${ }^{\mathrm{nn}}$ Also at Adiyaman University, Adiyaman, Turkey.

${ }^{\circ o}$ Also at The University of Iowa, Iowa City, Iowa, USA.

${ }^{\mathrm{pp}}$ Also at Mersin University, Mersin, Turkey.

${ }^{\mathrm{qq}} \mathrm{Also}$ at Kafkas University, Kars, Turkey.

${ }^{\mathrm{rr}}$ Also at Suleyman Demirel University, Isparta, Turkey.

${ }^{\mathrm{ss}}$ Also at Ege University, Izmir, Turkey.

${ }^{\text {tt} A l s o ~ a t ~ S c h o o l ~ o f ~ P h y s i c s ~ a n d ~ A s t r o n o m y, ~ U n i v e r s i t y ~ o f ~ S o u t h a m p t o n, ~ S o u t h a m p t o n, ~ U n i t e d ~ K i n g d o m . ~}$

uu Also at INFN Sezione di Perugia, Università di Perugia, Perugia, Italy.

${ }^{\mathrm{vv}}$ Also at University of Sydney, Sydney, Australia.

${ }^{w w}$ Also at Utah Valley University, Orem, Utah, USA.

${ }^{\mathrm{xx}}$ Also at Institute for Nuclear Research, Moscow, Russia.

${ }^{\mathrm{yy}}$ Also at University of Belgrade, Faculty of Physics and Vinca Institute of Nuclear Sciences, Belgrade, Serbia.

${ }^{z z}$ Also at Argonne National Laboratory, Argonne, Illinois, USA.

${ }^{\text {aaa }}$ Also at Erzincan University, Erzincan, Turkey.

${ }^{b b b}$ Also at Kyungpook National University, Daegu, Korea. 